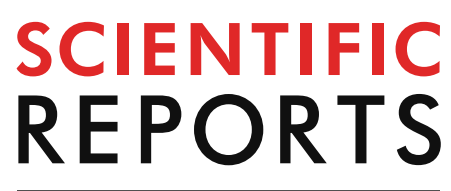

natureresearch

Check for updates

\title{
Genome-wide identification and characterization of $D C L$, AGO and RDR gene families in Saccharum spontaneum
}

\author{
Dong-Li Cui ${ }^{1}$, Jian-Yu Meng ${ }^{1}$, Xiao-Yan Ren ${ }^{2}$, Jing-Jing Yue ${ }^{3}$, Hua-Ying Fu ${ }^{1}$, Mei-Ting Huang ${ }^{1}$, \\ Qing-Oi Zhang ${ }^{2 \bowtie}$ \& San-Ji Gao ${ }^{1 \bowtie}$
}

RNA silencing is a conserved mechanism in eukaryotic organisms to regulate gene expression. Argonaute (AGO), Dicer-like (DCL) and RNA-dependent RNA polymerase (RDR) proteins are critical components of RNA silencing, but how these gene families' functions in sugarcane were largely unknown. Most stress-resistance genes in modern sugarcane cultivars (Saccharum spp.) were originated from wild species of Saccharum, for example S. spontaneum. Here, we used genomewide analysis and a phylogenetic approach to identify four $D C L, 21 A G O$ and $11 R D R$ genes in the $S$. spontaneum genome (termed SsDCL, SsAGO and SsRDR, respectively). Several genes, particularly some of the SSAGOs, appeared to have undergone tandem or segmental duplications events. RNAsequencing data revealed that four SsAGO genes (SsAGO18c, SsAGO18b, SsAGO10e and SsAGO6b) and three SsRDR genes (SSRDR2b, SsRDR2d and SSRDR3) tended to have preferential expression in stem tissue, while SsRDR5 was preferentially expressed in leaves. qRT-PCR analysis showed that SsAGO10c, SsDCL2 and SsRDR6b expressions were strongly upregulated, whereas that of SsAGO18b, SsRDR1a, SsRDR2b/2d and SsRDR5 was significantly depressed in S. spontaneum plants exposed to PEG-induced dehydration stress or infected with Xanthomonas albilineans, causal agent of leaf scald disease of sugarcane, suggesting that these genes play important roles in responses of $S$. spontaneum to biotic and abiotic stresses.

RNA silencing, also known as RNA interference (RNAi), plays an important role in multiple processes in plants, including growth and development, epigenetic modifications and responses to and defenses against abiotic and biotic stresses ${ }^{1-3}$. Several essential steps and core components of RNA silencing pathways are well-characterized. RNA silencing is initially triggered by the formation of double stranded RNAs (dsRNAs) that are subsequently cleaved by the RNase III-type DICER-LIKE proteins (DCL) into small RNA duplexes (sRNAs) of 21-24 nucleotides that include short-interfering RNAs (siRNAs) and microRNAs (miRNAs) ${ }^{4,5}$. Diverse sRNAs are denatured and incorporated into the multi-component RNA-induced silencing complex (RISC) having an Argonaute (AGO) protein at its catalytic core ${ }^{6,7}$. The RISC binds complementary mRNAs guided by single-stranded sRNAs to mediate processes such as translational inhibition, RNA degradation or chromosome modification ${ }^{8,9}$. These sRNAs are amplified from the targeted RNA by cellular host RNA-dependent RNA polymerases (RDRs) to produce additional dsRNAs that will be processed into secondary siRNAs that amplify the silencing signal ${ }^{10,11}$. Notably, RNA silencing-based immunity is also integrated with R gene-mediated immunity in plants for defense against pathogens ${ }^{12-14}$.

The proteins encoded by DCL, AGO, and RDR gene families are core components of the RNA silencing process ${ }^{5}$. DCLs contain a DEAD domain, a helicase conserved C-terminal (Helicase C) domain, a Dicer dimerization domain (Dicer dimer), a PAZ domain (PAZ), a Ribonuclease III domain (Ribonuclease 3), and a

\footnotetext{
${ }^{1}$ National Engineering Research Center for Sugarcane, Fujian Agriculture and Forestry University, Fuzhou 350002, Fujian, China. ${ }^{2}$ College of Agriculture, Fujian Agriculture and Forestry University, Fuzhou 350002, Fujian, China. ${ }^{3}$ FAFU and UIUC-SIB Joint Center for Genomics and Biotechnology, Fujian Provincial Key Laboratory of Haixia Applied Plant Systems Biology, Fujian Agriculture and Forestry University, Fuzhou 350002, Fujian, China. ${ }^{\circledR}$ email:000q010002@fafu.edu.cn; gaosanji@fafu.edu.cn
} 
double-stranded RNA-binding domain (DSRM) ${ }^{15,16}$. AGOs have four functional domains, i.e., variable MID and $\mathrm{N}$-terminal domains, and conserved PAZ and PIWI domains ${ }^{7,17}$. The PAZ domain can anchor sRNA duplexes with a two-nucleotide $3^{\prime}$ overhang via the specific binding pocket and the PIWI domain that has a similar fold to RNase $\mathrm{H}$ and exhibits endonuclease activity, thus playing an important role in target RNA cleavage ${ }^{18}$. RDRs share a special conserved RNA-dependent RNA polymerase (RdRP) catalytic domain ${ }^{19}$. The DCL, AGO, and RDR gene families in plants have species-dependent differences in gene numbers, which range from 20 genes in Arabidopsis $^{20}$ to 51 genes in Brassica species ${ }^{21}$. Notably, different members of DCL, AGO, and RDR families play different roles in RNA silencing in plants, but they also share partially redundant functions ${ }^{20}$. Currently, there is limited information about DCL, AGO, and RDR gene families in sugarcane.

Sugarcane is an important sugar crop that accounts for $80 \%$ of sugar production worldwide and also is one of the most sustainable energy crops that can serve as a biofuel source ${ }^{22,23}$. Modern sugarcane hybrids originated from crosses between S. officinarum and one or more other Saccharum species and their progenies were progressively backcrossed with different $S$. officinarum clones ${ }^{24-26}$. This process of recurrent introgressive hybridization contributed to commercial hybrids that are highly outcrossed, heterozygous polyploids ${ }^{26}$. Modern sugarcane cultivars offer high sugar content that can primarily be attributed to S. officinarum, whereas other traits (e.g., growth vigor, stress resistance, and ratooning) mainly arose from S. spontaneum ${ }^{27-29}$. Recently, a genome sequence of the sugarcane wild species clone AP85-441, a haploid S. spontaneum, was determined and assembled into 32 pseudo-chromosomes comprising eight homologous groups of four members each ${ }^{28}$. In addition, a BAC (bacterial artificial chromosome)-based monoploid genome sequence of cultivar R570 ${ }^{27}$ and a polyploid genome sequence of cultivar SP80-3280 ${ }^{30}$ were also sequenced and assembled. These sugarcane genomic sequences provide valuable reference sequences in the post-genomics era ${ }^{23}$.

Sugarcane often suffers diverse biotic (e.g. pathogenic microorganisms) and abiotic (e.g., drought, cold and high salinity) stresses ${ }^{22}$. Drought stress is one of the most important abiotic stress factors in sugarcane growth and yield worldwide, including in China ${ }^{31,32}$. Additionally, leaf scald caused by Xanthomonas albilineans is one of three main sugarcane bacterial diseases, and is responsible for significant loss in cane yield and juice quality ${ }^{33}$. Leaf scald exists in some sugarcane-producing areas in China, where it represents a potential threat to the sugar industry ${ }^{34,35}$. In this study we identified and classified protein members of the DCL, AGO and RDR gene families in the S. spontaneum genome, and analyzed the functions of these genes to understand their roles in responses to polyethylene glycol (PEG)-induced dehydration stress and X. albilineans infection. Our findings will provide important information for exploration of molecular resistance mechanisms in S. spontaneum.

\section{Methods}

Identification of putative DCL, AGO, and RDR genes in S. spontaneum. Hidden Markov Model (HMM) profiles of the characterized and conserved domains of DCL, AGO and RDR families were retrieved from the protein family database (Pfam, https://pfam.xfam.org//) to search for these gene families in the $S$. spontaneum genome database (https://www.life.illinois.edu/ming/downloads/Spontaneum_genome/) ${ }^{28}$. DCL, AGO, and RDR protein sequences were identified based on the HMM profiles using HMMER software with default parameters ${ }^{37}$ and a cut-off value of $0.01^{38}$. To ensure the complete identification of the three gene families, we further Blasted DCLs, AGOs and RDRs from maize genome sequences ${ }^{39}$ against the $S$. spontaneum genome database. Conserved domains in all candidate genes were examined using the Pfam and Simple Modular Architecture Research Tool (SMART, https://smart.embl-heidelberg.de/) program. Sequence length, molecular weight and the isoelectric point of DCL, AGO and RDR proteins were predicted using tools at the ExPasy website (https ://web.expasy.org/protparam/).

In-silico analysis of gene structure, promoter cis-acting elements and protein-protein interactions. Information concerning conserved domains among DCL, AGO and RDR genes in S. spontaneum, termed $S s D C L, S s A G O$ and $S s R D R$, including the domain name and position were obtained by SMART (https ://smart.embl-heidelberg.de/). The domain structure and exon-intron organization of the three gene families were analyzed using the online program Gene Structure Display Server (GSDS2.0: https://gsds.cbi.pku.edu. $\mathrm{cn})^{40}$. One kilobase $(\mathrm{kb})$ upstream region from the initial codon of each candidate gene in the three gene families was used to search cis-elements by the PlantCARE program (https://bioinformatics.psb.ugent.be/webtools/plant care/html/). Network analysis of protein-protein interactions (PPIs) among all identified SsDCLs, SsAGOs and SsRDRs was performed using STRING v11.0 (https://string-db.org/) and corresponding maize proteins as reference sequences ${ }^{41}$. The minimum required interaction score was 0.400 , corresponding to medium confidence.

Chromosomal localization and phylogenetic analysis. The physical locations of $S s D C L, S s A G O$, and $S s R D R$ genes were determined from the S. spontaneum genome database. The chromosomal positions of the three gene families were mapped using Circos software ${ }^{42}$. Gene duplication events were analyzed using the Multiple Collinearity Scan toolkit (MCScanX) with default parameters ${ }^{43}$. Multiple sequence alignments with the respective protein family from Arabidopsis ${ }^{21}$, rice ${ }^{44}$, maize ${ }^{39}$ and $S$. spontaneum were performed using the ClustalW program in MEGA 7.0 software ${ }^{45}$. Phylogenetic trees were constructed using a Neighbor-Joining (NJ) method with 1,000 bootstrap replications.

Plant materials and experimental treatments. Cuttings of S. spontaneum clone SES208 were provided by the Center for Genomics and Biotechnology, Fujian Agriculture and Forestry University (Fuzhou, China). Clone SES208 is an octoploid donor used to generate the haploid AP85-441 clone through anther cultures ${ }^{26}$. The cuttings were grown in a plant growth chamber under a $16 \mathrm{~h} / 8 \mathrm{~h}$ light/dark period at $30{ }^{\circ} \mathrm{C}$ and $70 \%$ relative humidity (RH). Four-week-old plants (three leaves fully expanded) of clone SES208 were used in two experi- 
mental treatments: drought stress and bacterial stress. Roots of 24 plants were immersed in a 25\% PEG-6000 solution for $0,3,6$, and $12 \mathrm{~h}$ and respective top young leaf samples were collected. Leaves from another 24 plants were inoculated with $X$. albilineans strain Xa-FJ1 following the protocol described by Lin et al. ${ }^{34}$ and inoculated leaves were collected at $0,24,48$, and $72 \mathrm{~h}$ post inoculation (hpi). All samples were frozen in liquid nitrogen and stored at $-80^{\circ} \mathrm{C}$ until total RNA was extracted.

Quantitative real-time PCR (qRT-PCR). Total RNA from leaf tissues was extracted by TRIzol reagent (Invitrogen/Life Technologies, Carlsbad, CA, USA) according to the manufacturer's instructions. RNA quality was analyzed by electrophoresis on a $1 \%$ agarose gel and RNA amounts were quantified using a Synergy ${ }^{\mathrm{ma}} \mathrm{H} 1$ hybrid multimode reader (BioTek, Winooski, VT, USA). All RNA samples were diluted to a working concentration of $1.0 \mu \mathrm{g} / \mu \mathrm{L}$ with RNase-free $\mathrm{H}_{2} \mathrm{O}$ for further analysis. Total RNA $(1.0 \mu \mathrm{g})$ was used in reverse transcription (RT) reactions to generate the first strand cDNA by HiScript II RT (Hongfeng Science and Technology, Nanjing, China) with random primers following the manufacturer's directions. Because of the high homology in some gene pairs, we could not design primers with high specificity for every gene members, thus nine, three, and six candidate genes from $S s A G O s, S s D C L s$, and $S s R D R s$, respectively, were chosen to represent the different sub-families for quantitative real time PCR (qRT-PCR) transcriptional expression analyses. These genes primer pairs were designed with the GenScript Real-time PCR (TaqMan) Primer Design tool (https://www.genscript. com/tools/real-time-pcr-taqman-primer-design-tool) (Table S1). The qRT-PCR reactions were performed using $94^{\circ} \mathrm{C}$ for $30 \mathrm{~s}$ followed by 40 cycles of $95^{\circ} \mathrm{C}$ for $10 \mathrm{~s}$ and $60^{\circ} \mathrm{C}$ for $30 \mathrm{~s}$. The housekeeping gene, glyceraldehyde 3-phoshate dehydrogenase (GAPDH), was used as an internal control for normalization. The qRT-PCR results were analyzed by the $2^{-\Delta \Delta \mathrm{Ct}}$ quantitative method to determine differences in gene expression ${ }^{44}$. Three biological and three technical replicates were carried out for each sample.

Data analysis. Relative expression levels determined from qRT-PCR data at different time points for each cultivar were analyzed using one-way ANOVA. Multiple comparisons of the means were conducted by the SNK (Student-Newman-Keuls) Test. All statistical analyses were carried using SAS version 8.1 (SAS Institute, Cary, NC, USA).

\section{Results}

Identification and structural analysis of SsAGO, SsDCL, and SsRDR genes. To identify the AGO, DCL, and RDR gene families in S. spontaneum, we gathered the previously characterized and conserved domains of the three gene families and used HMMER software to search for corresponding domains in the S. spontaneum genome database. In addition, members of the three gene families from maize genome sequences were blasted against the $S$. spontaneum genome database. After evaluation of the structural integrity of conserved domains and elimination of redundant sequences, four genes encoding DCL proteins (SsDCLs), 21 genes encoding AGO proteins (SsAGOs) and 11 genes encoding RDR proteins (SsRDRs) were identified in S. spontaneum (Table S2). The detailed characteristics of all genes identified in this study, including chromosomal location and protein properties (e.g., open reading frame (ORF) length, protein length (amino acid, aa), molecular weight (MW), and isoelectric point (IP)) are listed in Table 1.

The proteins encoded by the four identified SsDCLs ranged from 1,233 aa (SsDCL2, Sspon.01G0022370$1 \mathrm{~A})$ to 1,590 aa $(S s D C L 1 b$, Sspon.01G0001230-1A) and contained a Dicer_dimer domain, a PAZ domain, a Helicase_C domain, a DEAD domain, a RIBOc domain and one or two DSRM domains (one for SsDCL2/3, and two for SsDCL1a/b) (Fig. 1A). The 21 identified SsAGOs ranged from 628 aa (SsAGO10d, Sspon.08G0006580-4D) to 1,054 aa (SsAGO5a, Sspon.01G0028360-2B), and shared common domains including a DUF1785 domain, PAZ domain, PIWI domain, and ArgoN domain. ArgoL2 and ArgoMid domains that were present in most of the SsAGOs identified (14/21), but were absent in SsAGO2a/b, SsAGO3a/b/c, and SsAGO10c/d (Fig. 1B). SsRDRs varied from 683 aa for SsRDR4 (Sspon.05G0014430-4D) to 1,137 aa for SsRDR2c (Sspon.05G0009620-3C), which contained the common sequence motif for DNA-dependent RNA polymerases (RdRP) whereas SsRDR2a/b/c/d had another common sequence motif, the RRM domain (Fig. 1C). Comparative structural analysis for exonsintrons in the three gene families revealed that the number of introns ranged from two in $S s A G O 2 b / 3 a$ to 22 in SsAGO4, from 15 in SsDCL1a to 24 in SsDCL3, and from one in SsRDR6b to 17 in SsRDR3 (Figure S1).

Furthermore, pairwise sequence alignment and identity analysis revealed that the conversed motifs among these gene members in each DCL, AGO and RDR families were high discrepancy (Figure S2 and Table S3). The identifies of amino acid sequences in DCL, AGO and RDR gene families were $29.7-85.8 \%, 12.3-99.3 \%$ and $11.3-100 \%$, respectively (Table S3). Among gene pairs of alleles, these sequences shared higher identifies with each other. For example, the gene pairs of alleles in SsDCLs, the amino acid identity was $85.8 \%$ between SsDCL1a and $S s D C L 1 b$; the gene pairs of alleles in AGOs, the amino acid identities were 91.5\% ( $s$ sAGO2a and $S s A G O 2 b$ ), 83.6-91.8\% (SsAGO3a, SsAGO3b and SsAGO3c), 60.5-89.5\% (SsAGO5a, SsAGO5b, SsAGO5c, and SsAGO5d), 93.2\% (SsAGO6a and SsAGO6b), 43.5-97.3\% (SsAGO10a, SsAGO10b, SsAGO10c, SsAGO10d and SsAGO10e), and 53.0-95.2\% (SsAGO18a, SsAGO18b, SsAGO18c and $S s A G O 18 d)$. The gene pairs of alleles in RDRs, the amino acid identities were 100\% (SsRDR1a and $S s R D R 1 b$ ), 77.4-99.6\% (SsRDR2a, SsRDR2b, SsRDR2c and SsRDR2d) and $83.7 \%$ (SsRDR6a and $S s R D R 6 b)$.

Prediction of cis-acting elements in the putative gene promoters of SsAGO, SsDCL and SsRDR. Sequences $(1 \mathrm{~kb})$ of upstream of the translation initiation codon for the $S s A G O, S s D C L$, and $S s R D R$ genes were examined for the presence of cis-acting elements using the PLANTCARE online database. In addition to cis-acting elements that are characteristic of eukaryotic promoters, various cis-acting elements including those associated with plant growth, development, and stress responses were found among SsAGO, SsDCL, and 


\begin{tabular}{|c|c|c|c|c|c|c|c|c|}
\hline \multirow[b]{2}{*}{ Gene name } & \multirow[b]{2}{*}{ Gene ID } & \multicolumn{3}{|l|}{ Location } & \multicolumn{4}{|c|}{ Protein } \\
\hline & & Chromosome & Start & End & $\begin{array}{l}\text { CDS } \\
\text { (bp) }\end{array}$ & Length (aa) & Mw (Da) & PI \\
\hline \multicolumn{9}{|c|}{\begin{tabular}{|l} 
Dicer-like (DCL) \\
\end{tabular}} \\
\hline SsDCL1a & Sspon.01G0001230-3D & Chr1D & $3,692,925$ & $3,701,074$ & 4,377 & 1,458 & $163,204.71$ & 6.05 \\
\hline SsDCL1b & Sspon.01G0001230-1A & ChrlA & $3,976,165$ & $3,983,525$ & 4,770 & 1,590 & $178,065.62$ & 6.05 \\
\hline SsDCL2 & Sspon.01G0022370-1A & ChrlA & $81,960,057$ & $81,976,273$ & 3,699 & 1,233 & $139,512.97$ & 7.06 \\
\hline SsDCL3 & Sspon.01G0019860-4D & Chr1D & $71,661,374$ & $71,672,007$ & 4,752 & 1584 & $179,009.05$ & 6.24 \\
\hline \multicolumn{9}{|c|}{ Argonaute (AGO) } \\
\hline SsAGO2a & Sspon.05G0030950-2D & Chr5D & $11,880,179$ & $11,884,678$ & 3,066 & 1,021 & $111,963.65$ & 8.73 \\
\hline SsAGO2b & Sspon.05G0037340-1D & Chr5D & $11,924,233$ & $11,928,734$ & 2,763 & 921 & $100,646.66$ & 7.81 \\
\hline SsAGO3a & Sspon.05G0030960-1C & Chr5C & $2,396,093$ & $2,401,132$ & 3,048 & 1,015 & $110,049.24$ & 9.30 \\
\hline SsAGO3b & Sspon.05G0030950-1C & Chr5C & $2,386,282$ & $2,391,173$ & 2,904 & 967 & $104,620.95$ & 9.27 \\
\hline SsAGO3c & Sspon.05G0030950-1P & Chr5D & $12,513,102$ & $12,517,597$ & 3,036 & 1,011 & $109,860.1$ & 9.35 \\
\hline SsAGO4 & Sspon.02G0016200-2D & Chr3D & $39,536,146$ & $39,547,268$ & 2,838 & 946 & $105,523.71$ & 8.60 \\
\hline SsAGO5a & Sspon.01G0028360-2B & Chr1B & $96,848,274$ & $96,854,895$ & 3,165 & 1,054 & $115,793.38$ & 9.42 \\
\hline SsAGO5b & Sspon.01G0014460-3D & Chr1D & $87,130,069$ & $87,135,808$ & 2,742 & 913 & $102,643.41$ & 9.28 \\
\hline SsAGO5c & Sspon.01G0014460-1A & Chr1A & $41,213,733$ & $41,219,461$ & 2,418 & 806 & $90,379.42$ & 9.55 \\
\hline SsAGO5d & Sspon.01G0014460-2B & Chr1B & $96,914,953$ & $96,920,845$ & 2,784 & 927 & $103,595.76$ & 9.55 \\
\hline SsAGO6a & Sspon.07G0020900-1A & Chr7A & $78,332,899$ & $78,336,592$ & 1,902 & 634 & $70,983.26$ & 9.46 \\
\hline SsAGO6b & Sspon.07G0020900-2D & Chr7D & $60,984,388$ & $60,994,488$ & 2,070 & 689 & $77,218.74$ & 9.34 \\
\hline SsAGO10a & Sspon.08G0006580-1P & Chr8D & $17,872,226$ & $17,876,344$ & 2,271 & 757 & $83,975.06$ & 9.22 \\
\hline SsAGO10b & Sspon.08G0006580-1A & Chr8A & $20,403,262$ & $20,409,634$ & 2,829 & 942 & $105,347.29$ & 9.47 \\
\hline SsAGO10c & Sspon.08G0006580-2B & Chr8B & $18,085,409$ & $18,091,645$ & 2,394 & 797 & $88,300.59$ & 9.67 \\
\hline SsAGO10d & Sspon.08G0006580-4D & Chr8D & $17,765,548$ & $17,771,337$ & 1,884 & 628 & $71,264.01$ & 9.40 \\
\hline SsAGO10e & Sspon.08G0006580-3C & Chr8C & $9,379,029$ & $9,385,180$ & 2,916 & 971 & $108,586.36$ & 9.43 \\
\hline SsAGO18a & Sspon.02G0007830-1A & Chr2A & $22,748,211$ & $22,753,686$ & 3,099 & 1,032 & $113,076.36$ & 9.47 \\
\hline SsAGO18b & Sspon.01G0024300-1A & Chr1A & $87,229,183$ & $87,234,462$ & 2,382 & 793 & $88,734.95$ & 9.29 \\
\hline SsAGO18c & Sspon.01G0024300-3D & Chr1D & $84,919,502$ & $84,924,770$ & 2,364 & 787 & $88,118.01$ & 9.00 \\
\hline SsAGO18d & Sspon.02G0007830-2D & Chr2D & $16,391,380$ & $16,396,270$ & 2,754 & 918 & $100,666.07$ & 9.44 \\
\hline \multicolumn{9}{|c|}{\begin{tabular}{|l|} 
RNA dependent RNA Polymerase (RDR) \\
\end{tabular}} \\
\hline SsRDR1a & Sspon.04G0003980-2D & Chr4D & $12,916,500$ & $12,921,015$ & 3,324 & 1,107 & $126,692.84$ & 7.73 \\
\hline SsRDR1b & Sspon.04G0003980-1A & Chr4A & $12,309,373$ & $12,314,472$ & 3,348 & 1,115 & $127,601.91$ & 7.53 \\
\hline SsRDR2a & Sspon.05G0009620-1A & Chr5A & $27,714,217$ & $27,719,638$ & 3,414 & 1,137 & $126,512.75$ & 6.67 \\
\hline SsRDR2b & Sspon.05G0009620-2B & Chr5B & $22,682,674$ & $22,688,038$ & 3,315 & 1,104 & $122,789.35$ & 6.50 \\
\hline SsRDR2c & Sspon.05G0009620-3C & Chr5C & $19,106,947$ & $19,112,258$ & 3,414 & 1,137 & $126,557.88$ & 6.39 \\
\hline SsRDR2d & Sspon.05G0009620-4D & Chr5D & $30,160,562$ & $30,165,865$ & 2,967 & 989 & $110,364.29$ & 7.29 \\
\hline SsRDR3 & Sspon.05G0014430-3C & Chr5C & $48,023,413$ & $48,044,408$ & 2076 & 691 & $79,290.78$ & 7.29 \\
\hline SsRDR4 & Sspon.05G0014430-4D & Chr5D & $52,146,251$ & $52,168,613$ & 2049 & 683 & $77,901.79$ & 6.25 \\
\hline SsRDR5 & Sspon.03G0023160-2C & Chr3C & $89,299,759$ & $89,307,281$ & 2,514 & 838 & $96,153.93$ & 8.70 \\
\hline SsRDR6a & Sspon.08G0019290-1B & Chr8B & $11,200,927$ & $11,205,278$ & 3,240 & 1,079 & $120,677.59$ & 7.10 \\
\hline SsRDR6b & Sspon.08G0019290-2D & Chr8D & $11,219,834$ & $11,222,839$ & 3,006 & 1,001 & $111,860.59$ & 8.24 \\
\hline
\end{tabular}

Table 1. Structural characteristics and physio-chemical properties of dicer-like (DCL), argonaute (AGO) and RNA dependent RNA polymerase (RDR) genes from $S$. spontaneum. CDS coding sequence, $M W$ molecular weight, $I P$ isoelectric point.

SsRDR family members. The upstream sequences of all $S s A G O, S s D C L$, and $S s R D R$ genes had the cis-acting element of eukaryotic promoters (CAAT-box) and, except for $S s A G O 2 b / 10 d$ and $S s R D R 2 a / 2 b / 6 b$, had a TATA-box that is another cis-acting element of eukaryotic promoters (Table S4). Numerous cis-acting elements associated with drought/dehydration response including DRE core motifs, MYB recognition or binding sites, MBS, MYC, and ABRE elements were present (Fig. 2). Genes including SsDCL3, SsAGO2b, SsAGO3c, SsAGO10c/d/e, $S s A G O 18 b, S s R D R 2 b$, and $S s R D R 4$ had more than ten cis-elements related to dehydration response. More than three classes of MYC elements were found in SsDCL2, SsAGO3a/b/c, SsAGO18a, SsRDR1a/b, SsRDR2d, and $S R D R 3$, but no MYB elements were predicted in $S s A G O 5 b$, SsAGO5d, and SsRDR6b.

Multiple cis-acting elements involved in plant wound and pathogen response were also predicted in these promoters. More than one cis-acting element (W box, TGACG-motif or TCA-element) related to salicylic acid (SA) response were present in all gene family members, except for SsAGO6a/b. Two or more cis-acting element 
a

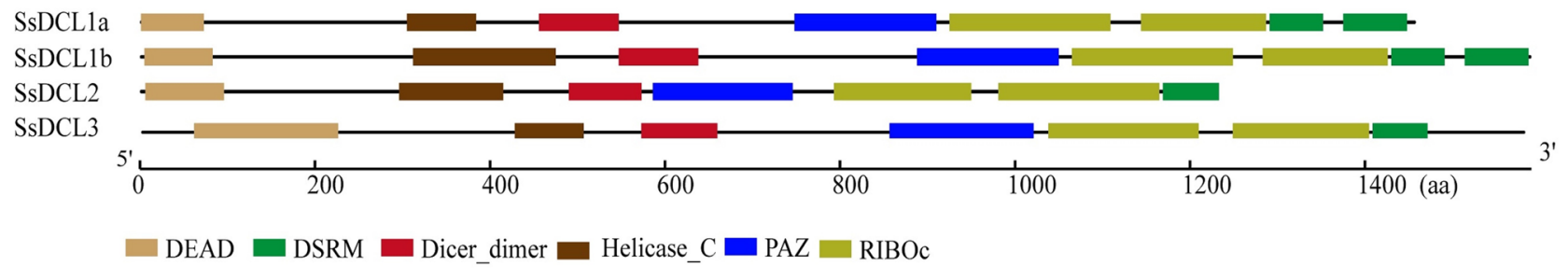

b

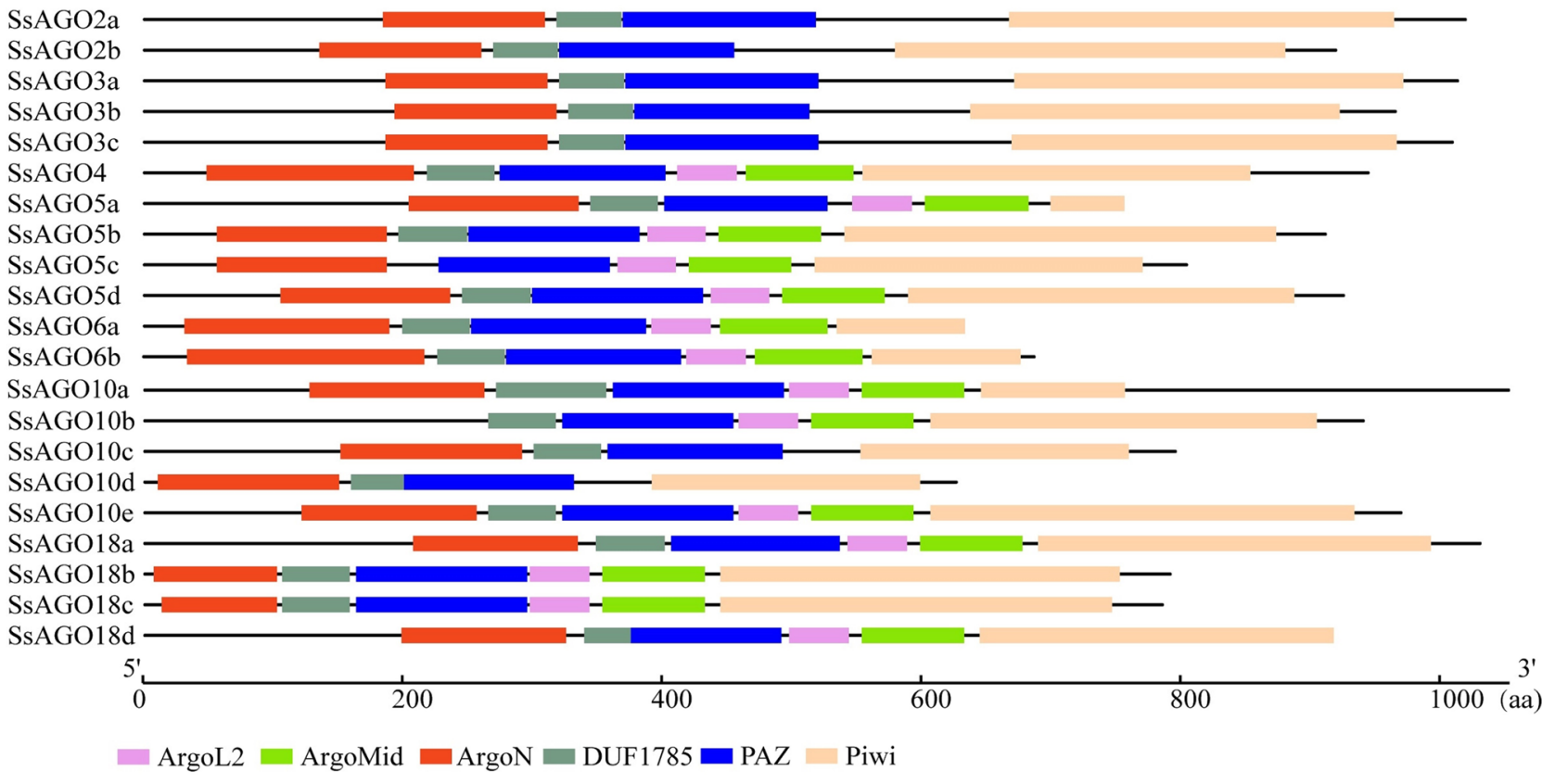

c

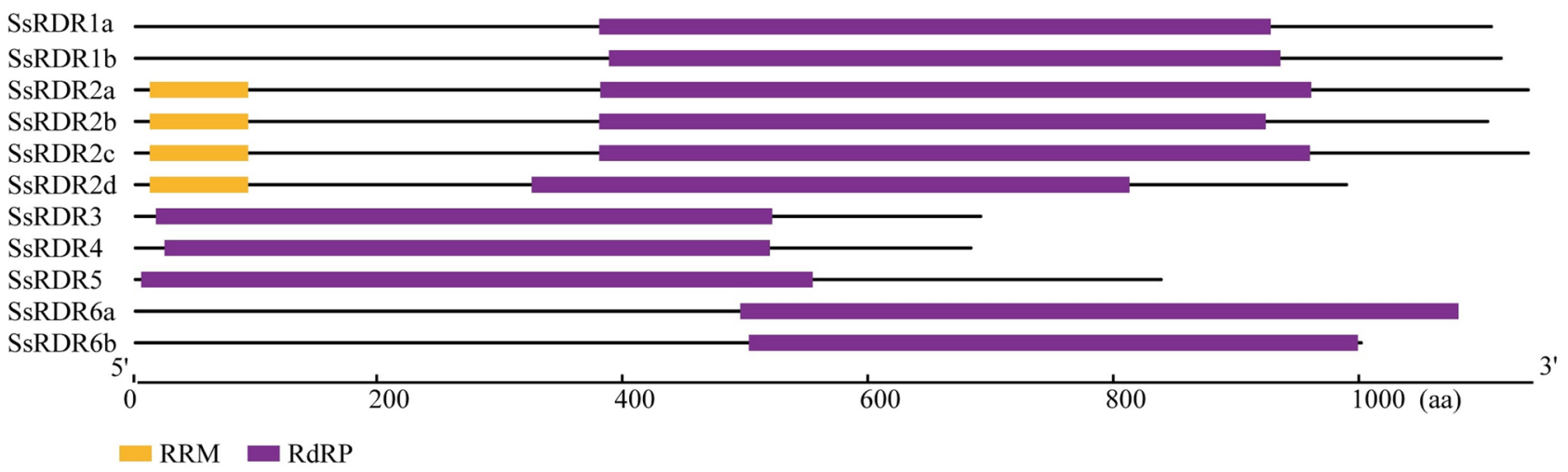

Figure 1. Structural domains of dicer-like protein (SsDCL) (a), argonaute protein (SsAGO) (b), and RNAdependent RNA polymerase (SsRDR) (c) from S. spontaneum. Domains are indicated by colored boxes. The scale bars at the bottom represent the length of proteins in aa (amino acid).

sites (CGTCA motif or TGACG motif) related to Methyl Jasmonate (MeJA) regulation presented in 24 promoters among the three gene families. Notably, the cis-element $\mathrm{W}$ box, which is related to responses to plant pathogen invasion, was present in SsDCL2, SsAGO2b, SsAGO3c, SsAGO5a/b/c/d, SsAGO10a/b/c/e, SsRDR1a/b, SsRDR3, SsRDR4, and SsRDR6b. The TC-rich repeat associated with plant defense against pathogen infection was present in $S s A G O 3 a$. Meanwhile, the WUN-motif related to mechanical damage was predicted only in the SsAGO6a/b promoter. 
drought

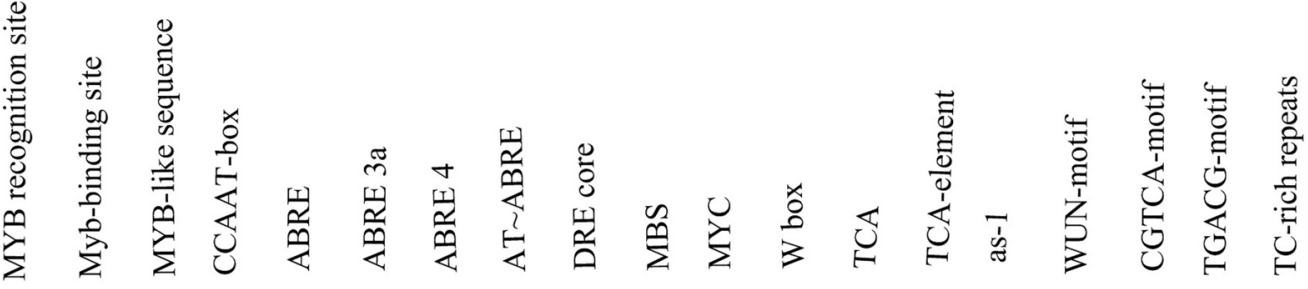

SsDCL1a

SsDCL1b

SsDCL2

SsDCL 3

SsAGO2a

SsAGO2b

SsAGO3a

SsAGO3b

SsAGO3c

SsAGO4

SsAGO5a

SsAGO5b

SsAGO5c

SsAGO5d

SsAGO6a

SsAGO6b

SsAGO10a

SsAGO10b

SsAGO10c

SsAGO10d

SsAGO10e

SsAGO18a

SsAGO18b

SsAGO18c

SsAGO18d

SsRDR1a

SsRDR1b

SsRDR2a

SsRDR2b

SsRDR2c

SsRDR2d

SsRDR3

SsRDR4

SsRDR5

SsRDR6a

SsRDR6b

\begin{tabular}{|c|c|c|c|c|c|c|c|c|c|c|c|c|c|c|c|c|c|c|}
\hline 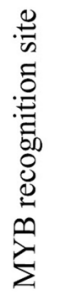 & 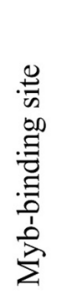 & 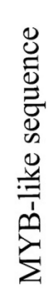 & 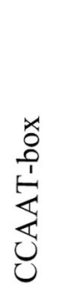 & 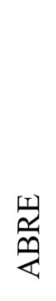 & $\begin{array}{l}\pi \\
m \\
0 \\
\frac{N}{n} \\
\end{array}$ & $\begin{array}{l}+ \\
\frac{1}{2} \\
\frac{0}{4}\end{array}$ & $\begin{array}{l}\frac{1}{0} \\
\frac{2}{4} \\
\frac{1}{4}\end{array}$ & 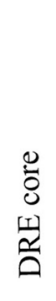 & $\stackrel{\tilde{n}}{\tilde{\Sigma}}$ & 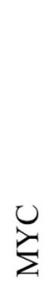 & $\begin{array}{l}0_{0}^{x} \\
3\end{array}$ & $\bigcup_{\forall}^{\mathbb{U}}$ & 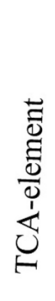 & $\begin{array}{l}\overline{\dot{g}} \\
\dot{g}\end{array}$ & 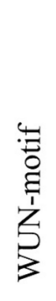 & 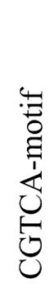 & 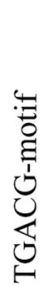 & 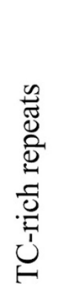 \\
\hline 0 & 1 & 0 & 0 & 3 & 0 & 0 & 0 & 0 & 0 & 3 & 0 & 0 & 1 & \begin{tabular}{|l|}
1 \\
\end{tabular} & 0 & 1 & 1 & 0 \\
\hline 0 & 0 & 0 & 1 & 1 & 1 & 1 & 0 & 0 & 2 & 0 & 0 & 0 & 0 & 2 & 0 & 0 & 0 & 0 \\
\hline 1 & 1 & 1 & 0 & 0 & 0 & 0 & 0 & 1 & 1 & 5 & 1 & 0 & 2 & 1 & 0 & 2 & 2 & 0 \\
\hline 1 & 0 & 1 & 0 & 7 & 0 & 0 & 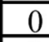 & 1 & 1 & 2 & ( & 0 & 1 & 0 & 0 & 1 & 1 & 0 \\
\hline 1 & 0 & 1 & 0 & 0 & 0 & 0 & 0 & 0 & 0 & 0 & $\overline{0}$ & 0 & 1 & 1 & 0 & 1 & 1 & 0 \\
\hline 0 & 0 & 0 & 1 & 8 & 1 & 1 & 1 & 4 & 0 & 1 & 1 & 1 & 4 & 0 & 0 & 4 & 4 & 0 \\
\hline 1 & 1 & 1 & 0 & 0 & 0 & 0 & 0 & 0 & 0 & 0 & 0 & 0 & 1 & 0 & 0 & 1 & 1 & 1 \\
\hline 1 & 0 & 1 & 0 & 0 & 0 & 0 & 0 & 0 & 0 & 0 & $\sqrt{2}$ & 0 & 1 & 0 & 0 & 1 & 1 & 0 \\
\hline 0 & 0 & 0 & 1 & 8 & 1 & 1 & 1 & 4 & 0 & 1 & 1 & 1 & 4 & 0 & 0 & 4 & 4 & 0 \\
\hline 0 & 0 & 0 & 1 & 0 & 0 & 0 & 0 & 1 & 4 & 4 & 0 & 0 & 0 & 2 & 0 & 0 & 0 & 0 \\
\hline 0 & 2 & 0 & 1 & 1 & 1 & 1 & 0 & 0 & 0 & 2 & 1 & 0 & 2 & 0 & 0 & 2 & 2 & 0 \\
\hline 0 & 0 & 0 & 0 & 2 & 0 & 0 & 0 & 0 & 1 & 1 & 1 & 0 & 2 & 1 & 0 & 2 & 2 & 0 \\
\hline 0 & 0 & 0 & 0 & 2 & 0 & 0 & 0 & 0 & 1 & 0 & 1 & 0 & 3 & 1 & 0 & 3 & 3 & 0 \\
\hline 0 & 0 & 0 & 0 & 2 & 0 & 0 & 0 & 0 & 1 & 0 & 1 & 0 & 2 & 1 & 0 & 2 & 2 & 0 \\
\hline 1 & 2 & 1 & 4 & 1 & 0 & 0 & 0 & 0 & 0 & 0 & 0 & 0 & 0 & 0 & 1 & 0 & 0 & 0 \\
\hline 1 & 2 & 1 & 4 & 1 & 0 & 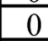 & 0 & 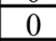 & 0 & 0 & 0 & 0 & 0 & 0 & 1 & 0 & 0 & 0 \\
\hline 0 & 0 & 0 & 1 & 1 & 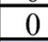 & 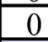 & 0 & 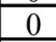 & 0 & 4 & 1 & 1 & 0 & 0 & 0 & 0 & 0 & 0 \\
\hline 0 & 1 & 0 & 1 & 1 & 1 & 1 & 0 & 0 & 0 & 5 & 2 & 0 & 0 & 0 & 0 & 0 & 0 & 0 \\
\hline 2 & 1 & $\frac{\pi}{2}$ & 0 & 1 & 1 & 1 & 0 & 0 & 0 & 3 & $\frac{2}{2}$ & 0 & 0 & 0 & 0 & 0 & 0 & 0 \\
\hline 1 & 0 & 1 & 1 & $\frac{1}{5}$ & $\frac{1}{2}$ & $\frac{1}{2}$ & $\frac{1}{1}$ & 2 & 0 & 3 & 20 & 0 & 2 & 0 & 0 & 2 & 2 & 0 \\
\hline 2 & 1 & $\frac{1}{2}$ & 0 & 1 & 1 & 1 & 0 & 0 & 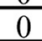 & 3 & 2 & 0 & 0 & 0 & 0 & 0 & 0 & 0 \\
\hline 0 & 1 & 0 & 1 & 0 & 0 & 0 & 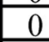 & 0 & 0 & 5 & 0 & 1 & 1 & 2 & 0 & 1 & 1 & 0 \\
\hline 0 & 1 & 0 & 2 & 3 & 1 & 1 & 0 & 0 & 1 & 2 & 0 & 0 & 4 & 1 & 0 & 4 & 4 & 0 \\
\hline 0 & 1 & 0 & 2 & 3 & 1 & 1 & 0 & 0 & 1 & 1 & 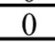 & 0 & 4 & 0 & 0 & 4 & 4 & 0 \\
\hline 0 & 1 & 0 & 1 & 1 & 0 & 0 & 0 & 0 & 0 & 4 & 0 & 1 & 1 & 1 & 0 & 1 & 1 & 0 \\
\hline 0 & 1 & 0 & 0 & 0 & 0 & 0 & 0 & 0 & 1 & 1 & 1 & 2 & 0 & 0 & 0 & 0 & 0 & 0 \\
\hline 0 & 1 & 0 & 0 & 0 & 0 & 0 & 0 & 0 & 1 & 1 & 1 & 2 & 0 & 0 & 0 & 0 & 0 & 0 \\
\hline 1 & 0 & 1 & 1 & 5 & 0 & 0 & 0 & 1 & 0 & 3 & 0 & 0 & 4 & 0 & 0 & 4 & 4 & 0 \\
\hline 1 & 0 & 1 & 1 & 4 & $\underline{0}$ & $\underline{0}$ & 0 & 1 & 0 & 3 & 0 & 0 & 5 & 0 & 0 & 5 & 5 & 0 \\
\hline 0 & 1 & $\frac{1}{0}$ & $\frac{1}{2}$ & $\frac{7}{4}$ & 0 & 0 & 0 & 1 & 0 & $\frac{3}{2}$ & $\sqrt{0}$ & 0 & $\frac{3}{4}$ & 0 & 0 & $\frac{\pi}{4}$ & $\frac{\pi}{4}$ & 0 \\
\hline 0 & $\frac{1}{0}$ & 0 & 1 & 0 & 0 & 0 & 0 & $\frac{1}{2}$ & 0 & 0 & 0 & 0 & 1 & 0 & 0 & 1 & 1 & 0 \\
\hline 1 & 1 & 1 & $\frac{1}{1}$ & 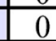 & 0 & $\frac{v}{0}$ & 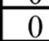 & 0 & 0 & $\frac{1}{2}$ & 1 & 0 & 0 & 0 & 0 & 0 & 0 & 0 \\
\hline 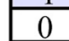 & 1 & 0 & 1 & $\frac{1}{2}$ & 1 & 1 & 1 & 1 & 1 & 3 & 2 & 0 & 1 & 0 & 0 & 1 & 1 & 0 \\
\hline 0 & 0 & 0 & 1 & 1 & 0 & 0 & 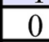 & 0 & 1 & 1 & 2 & 0 & 2 & 1 & 0 & 2 & 2 & 0 \\
\hline 0 & 0 & 0 & 1 & 1 & 1 & 1 & 0 & 0 & 0 & 1 & 0 & 0 & 0 & 1 & 0 & 0 & 0 & 0 \\
\hline 0 & 0 & 0 & 0 & 3 & 0 & 0 & 0 & 5 & 0 & 1 & 1 & 0 & 4 & 0 & 0 & 4 & 4 & 0 \\
\hline
\end{tabular}

Figure 2. Analysis of putative cis-acting elements related to response to drought or wound and pathogen stresses in S. spontaneum promoter sequences (1 kb) of SsDCL, SsAGO, and SsRDR genes. Numbers of elements present are indicated with darker blue shading representing higher numbers.

Phylogenetic analysis and chromosomal localization. To demonstrate phylogenetic relationships among identified genes encoding AGO, DCL, and RDR proteins, Neighbor-joining based phylogenetic trees were constructed using MEGA7 software based on the proteins of the three families from Arabidopsis, rice, maize, and S. spontaneum (Fig. 3, Table S2). All 19 DCL genes analyzed were clustered into four Clades, termed Clade I-IV (DCL1-4). Among the four SsDCL genes from S. spontaneum, SsDCL1a/1b genes were in Clade I, whereas SsDCL2 and SsDCL3 were grouped in Clade II and III, respectively. Unexpectedly, no SsDCL gene was found in Clade IV. All 59 AGO genes analyzed were clustered into three major Clades, including Clade I (AGO1/5/10), Clade II (AGO2/3/7), Clade III (AGO4/6/8/9). It is noteworthy that an AGO18 group from rice, maize and S. spontaneum falls into the Clade I. All 27 RDR genes were separated into four Clades, namely Clade I (RDR1), Clade II (RDRII), Clade III (RDR3/4/5), and Clade IV (RDR6). The 11 SsRDR genes from S. spontaneum were present in each of the four Clades.

All 36 identified S. spontaneum genes encoding DCLs, AGOs, and RDRs were precisely located on 19 of 32 S. spontaneum chromosomes (comprising 8 homologous groups of 4 members each): six genes on chromosome $5 \mathrm{D}$, four genes on chromosome $1 \mathrm{~A}, 1 \mathrm{D}$ and $5 \mathrm{C}$, three genes on chromosome $8 \mathrm{D}$, two genes on chromosome $1 \mathrm{~B}$, and one gene on the other remaining chromosomes $(1 \mathrm{C}, 2 \mathrm{~A}, 2 \mathrm{D}, 3 \mathrm{C}, 4 \mathrm{~A}, 4 \mathrm{D}, 5 \mathrm{~A}, 5 \mathrm{~B}, 7 \mathrm{~A}, 7 \mathrm{D}, 8 \mathrm{~A}, 8 \mathrm{~B}$, and $8 \mathrm{C}$ 

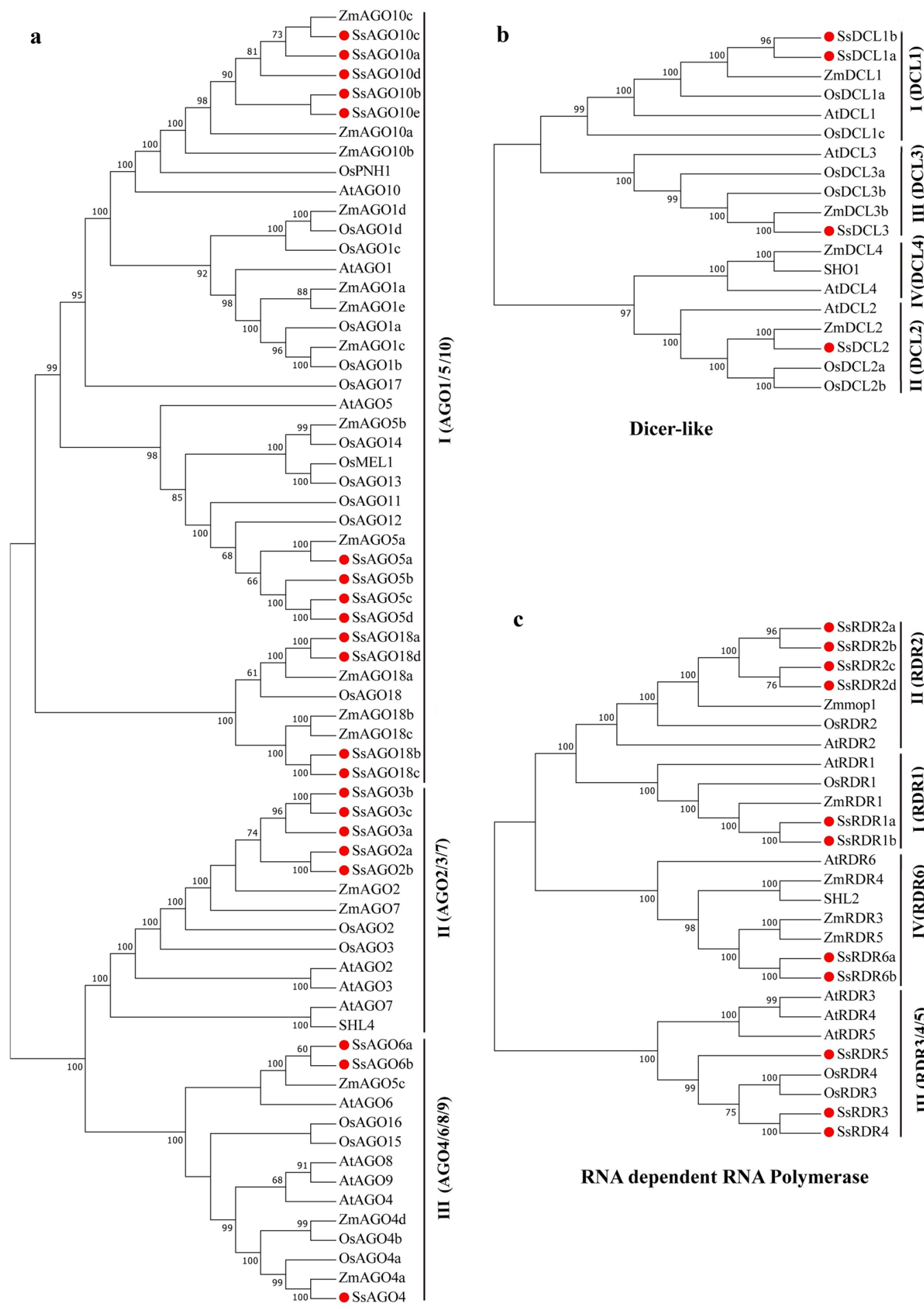

Dicer-like

\section{c}

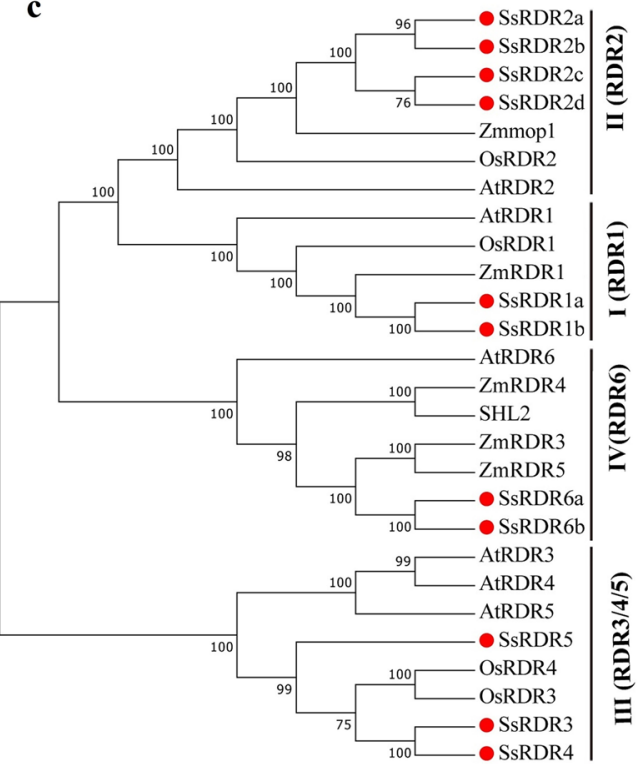

RNA dependent RNA Polymerase

Argonaute

Figure 3. Phylogenetic analysis of S. spontaneum SsAGO (a), SsDCL (b), and SsRDR (c) genes. NeighborJoining (NJ) trees were constructed using MEGA 7 software based on the protein sequences for each family member. Bootstrap support values from 1,000 replications are indicated above the branches. S. spontaneum genes are indicated by a red circle.

(Fig. 4). Of the three gene families, $S s A G O$ genes were widely distributed over thirteen chromosomes, followed by $S s R D R$ genes that were distributed over eight chromosomes (3C, 4A, 4D, 5A, 5B, 5C, 5D, 8B, and 8D), and SsDCL genes over three chromosomes (1A, 1C, and 1D). Meanwhile, duplication events that occurred over 


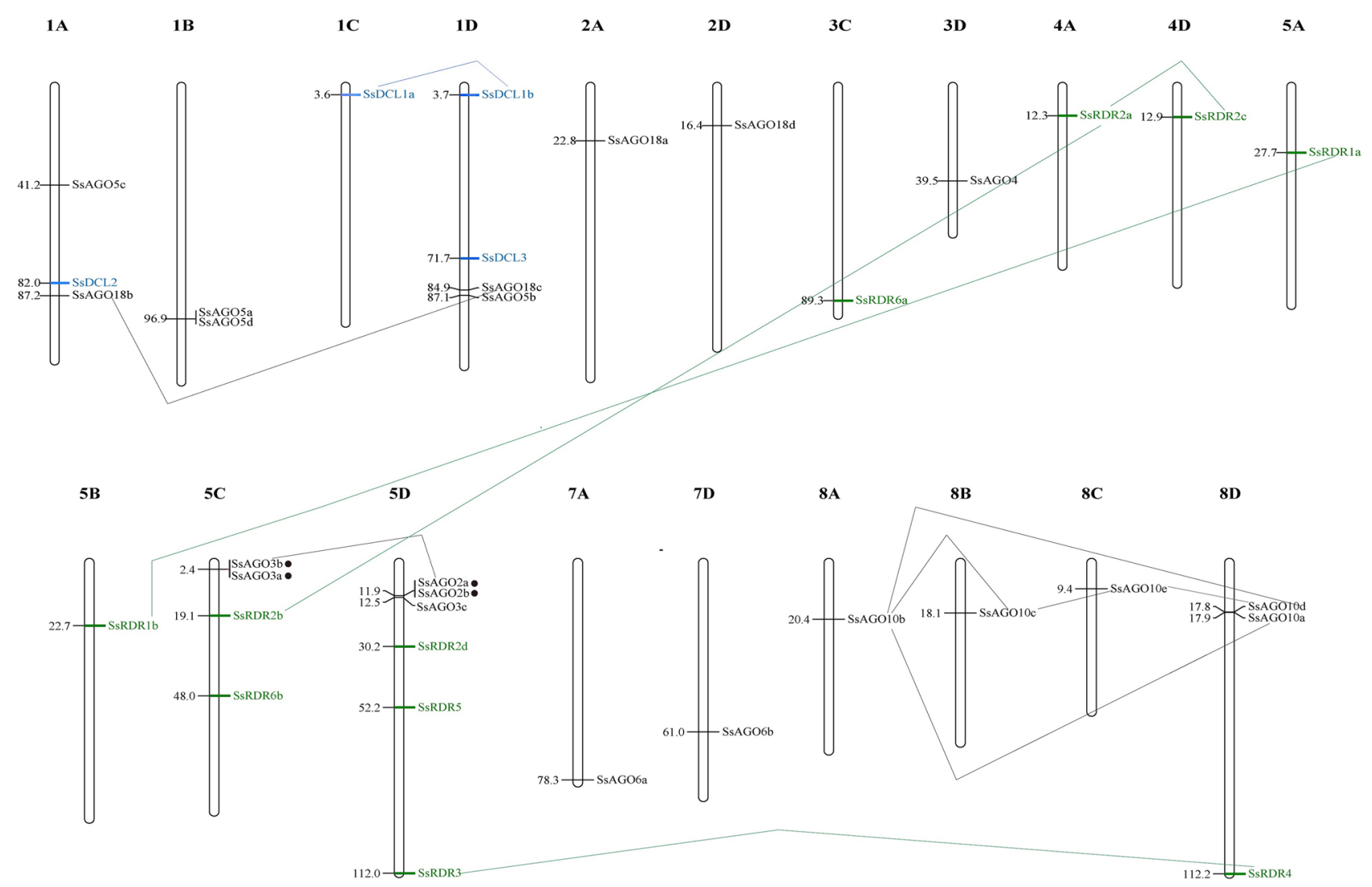

Figure 4. Chromosome localization of $S s D C L$ (in blue), $S s A G O$ (in black), and $S s R D R$ (in green) genes. The chromosome number is shown at the top of each bar. Horizontal bars represent the gene locations on each chromosome with positions in $\mathrm{Mb}$ (megabases) shown. Genes having tandem duplications are indicated by solid circles, whereas segmental duplication genes are joined by blue (SsDCLs), black (SsAGOs), and green lines (SsRDRs).

the course of $S$. spontaneum genome evolution were revealed by analysis with the BLASTp tool and MCScanX software. Two pairs of tandem duplicated SsAGO genes ( $S s A G O 2 a$ vs $S s A G O 2 b$; SsAGO3a vs SsAGO3b), which localized to chromosomes 5C and 5D, respectively, shared $91.5 \%$ and $83.6 \%$ identity with each other at an amino acid level. No tandem duplicated genes were seen for SsDCLs and SsRDRs. Twelve pairs of segmental duplicated genes were also found among the three families (one pair in SsDCLs, seven pairs in SsAGOs, and four pairs in SsRDRs), indicating that some of these genes could have been generated by gene duplication and that segmental duplication events play a major role in $S$. spontaneum genome evolution.

Protein-protein interaction networks for SsAGOs, SsDCLs, and SsRDRs. To investigate proteinprotein interactions (PPIs) among the 36 proteins (4 SsDCLs, 21 SsAGOs, and 11 SsRDRs) identified for $S$. spontaneum, a PPI network was predicted in-silico with the STRING database using maize sequences as queries. PPI network analysis showed that 20 of 36 identified proteins interacted with each other, including SsDCL1b, SsDCL2, SsDCL3, SsAGO3a, SsAGO4, SsAGO5a/d, SsAGO6a/b, SsAGO10a/c, SsAGO18a/b, SsRDR1a, SsRDR2b/d, SsRDR3, SsRDR/4, and SsRDR6a/b (Fig. 5). Some of these proteins (SsDCL1b-SsAGO6aSsRDR2b/d, SsDCL1b-SsAGO18a-SsRDR2b/d, SsDCL1b-SsAGO6a-SsRDR6a/b, SsDCL1b-SsAGO10aSsRDR6a/b, SsDCL2-SsAGO3a-SsRDR2b/d, SsDCL3-SsAGO4-SsRDR2b/d, SsDCL3-SsAGO6a-SsRDR2b/d, and SsDCL3-SsAGO18b-SsRDR2b/d) interacted strongly. These results indicated that various combinations of three core components of SsDCLs, SsAGOs, and SsRDRs may participate in different RNA silencing pathways in S. spontaneum.

Expression pattern of SsAGOs, SsDCLs and SsRDRs in leaf and stem tissues. To determine the temporal and spatial expression patterns of genes encoding AGO, DCL, and RDR in S. spontaneum, transcriptional expression analysis was performed in leaf and stem samples from seedlings as well as from pre-mature and mature plants using the RNA-sequencing (RNA-seq) database (https://www.life.illinois.edu/ming/downloads/ Spontaneum_genome/) (Fig. 6). Overall, no obvious temporal expression pattern was observed among the 36 identified genes, but expression patterns that tended to be specific to some tissues were observed. The four genes encoding SsDCLs exhibited no or very weak expression levels in either leaf or stem tissue. For the SsAGO genes, five genes ( $S s A G O 3 b, S s A G O 18 c$, SsAGO18b, SsAGO10e, and SsAGO6b) had high expression levels in both leaf and stem at all growth stages whereas, except for the $S s A G O 3 b$, showed higher expression levels in stem tissues 


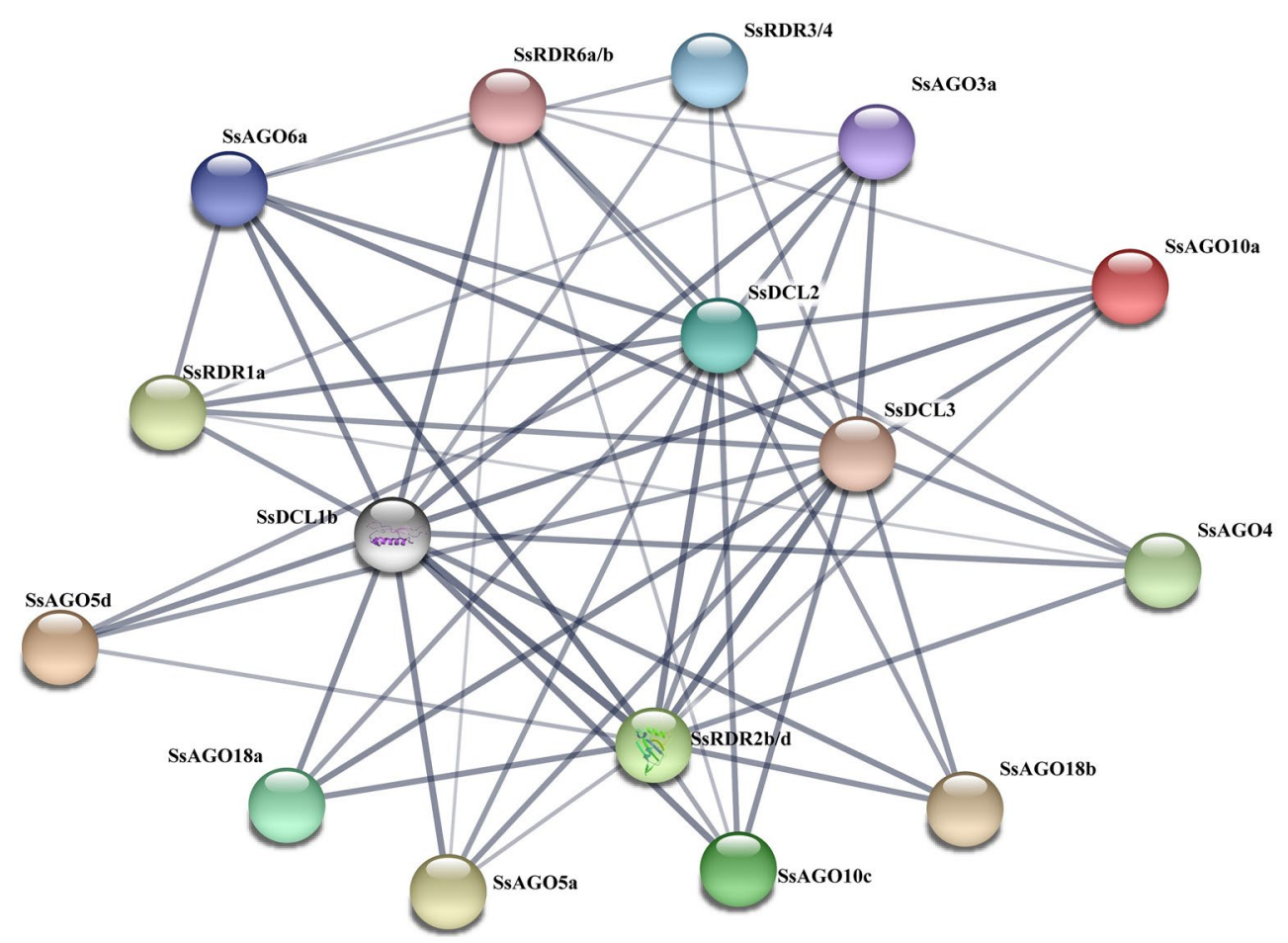

Figure 5. Schematic representation of protein-protein interaction (PPI) networks between SsDCLs, SsAGOs, and SsRDRs from S. spontaneum. Nodes having different colors indicate different proteins. Gray lines connect proteins within the PPI networks with darker colors and thicker lines indicating higher core PPI values.

relative to leaf tissues. The $S s A G O 18 a$ gene was also preferentially highly expressed in stem tissues, but not in leaf tissues, particularly mature or pre-mature leaves. The $S s A G O 10 c$ gene had moderate expression levels, but the others were absent or had very weak expression levels in leaves and stems. Of the $S s R D R$ genes, four ( $S s R D R 2 b$, $S s R D R 2 d, S s R D R 3$, and SsRDR5) exhibited higher expression levels in leaves and stems, whereas another five (SsRDR1a/b, SsRDR2c, SsRDR4, and SsRDR6a) had no detectable expression in either tissue type. Notably, of the four high-expression genes, $S s R D R 5$ had higher expression levels in leaves compared to stems. Conversely, $S s R D R 2 b, S s R D R 2 d$, and $S s R D R 3$ genes had higher expression in stems relative to leaves.

SsAGO, SsDCL, and SsRDR expression patterns induced by polyethylene glycol (PEG)-induced dehydration stress. To measure SsAGO, SsDCL, and SsRDR expression patterns in S. spontaneum under dehydration stress, the transcript expression for 18 candidate genes (9, 3 and 6 SsAGOs, SsDCLs, and SsRDRs, respectively) were analyzed by qRT-PCR upon the young plants of $S$. spontaneum clone SES208 subjected to PEG6000 treatment for 0-12 h(Fig. 7). The expression levels of five SsAGO genes (SsAGO2b, SsAGO5a, SsAGO5c, $S s A G O 6 b$, and $S s A G O 10 c$ ) in top young leaves were significantly upregulated by $>2.5$-fold, while $S s A G O 5 a$, $S s A G O 5 c$, and $S s A G O 10 c$ had particularly large increases of 18-, 11- and 35-fold, respectively, after $12 \mathrm{~h}$ of PEG treatment. Meanwhile, expression of $S s A G O 18 b$ was significantly downregulated after PEG treatment for 6-12 h. In the SsDCL family, SsDCL1a expression was significantly upregulated by PEG treatment for 3-6 h with an increase of twofold, whereas transcript levels of SsDCL2 and SsDCL3 were dramatically increased by 3-ninefold at 3-12 h and 10-25-fold at 6-12 h, respectively, post PEG treatment. In the RDR family, SsRDR3 and SsRDR6b expression level were highly upregulated with increases of 6-ninefold and 2-threefold, respectively, under dehydration stress (3-12 h). Additionally, SsRDR1a and SsRDR5 expression levels were significantly depressed whereas that of the $S s R D R 2 b$ gene did not significantly change.

Response of SsAGO, SsDCL and SsRDR expression to Xanthomonas albilineans infection. Expression profiles for the 18 candidate genes were also assessed by qRT-PCR on the young leaves of S. spontaneum clone SES208 inoculated with X. albilineans. Seven of the SsAGO candidate genes had decreased expression levels upon X. albilineans infection, with $S s A G O 5 c$, SsAGO10e, and $S s A G O 18 b$ showing significant down-regulation (Fig. 8). SsAGO10c expression was highly upregulated with increases of 5-13-fold at 48-72 hpi. Expression of the $S s A G O 6 b$ gene varied, with significant decreases at 24 and 72 hpi but increases at 48 hpi. Of the three $S s D C L$ genes tested, expression of SsDCL1a was dramatically depressed at $72 \mathrm{hpi}$, whereas that of SsDCL2 and $S s D C L 3$ was highly upregulated with increases of 3-4 folds at 48-72 hpi and $\sim$ twofold at 72 hpi, respectively. Five $S s R D R$ genes were downregulated to some extent by $X$. albilineans infection, but $S s R D R 6$ was significantly depressed at $48 \mathrm{hpi}$ and highly upregulated (tenfold increase) at $72 \mathrm{hpi}$. 


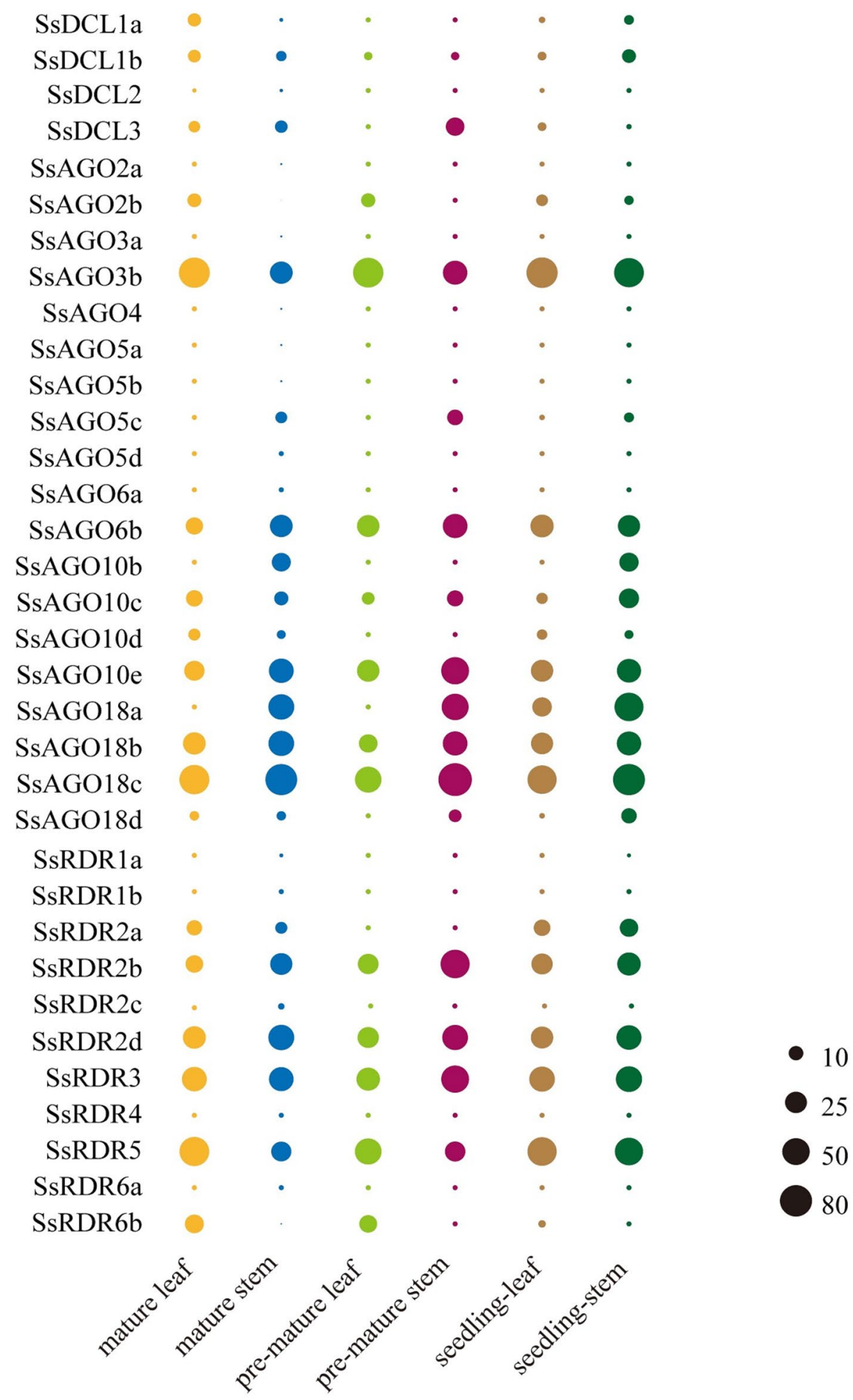

Figure 6. Heat map showing spatiotemporal expression patterns of genes encoding $S s D C L$, SsAGO, and $S s R D R$ in various $S$. spontaneum tissues including mature leaf, mature stem, pre-mature leaf, pre-mature stem, seedling leaf, and seedling stem. The size of the circles represents normalized expression level wherein larger circles correspond to higher expression levels.

\section{Discussion}

Modern sugarcane cultivars originated from nobilization processes have contributed to worldwide advances in the sugar industry over the last century ${ }^{25}$. Modern sugarcane cultivars have complex genomes that present major 


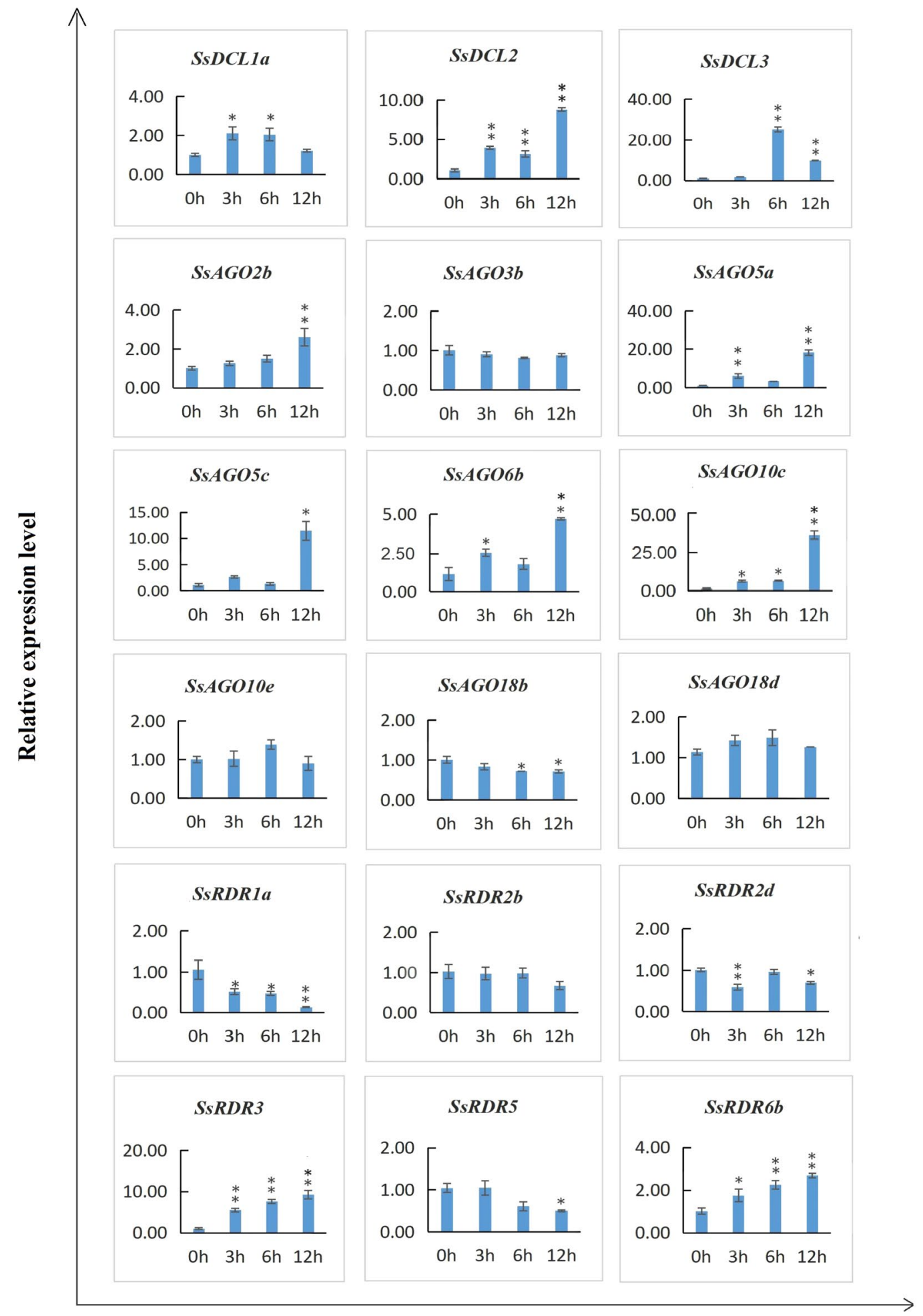

Figure 7. Quantitative real time PCR (qRT-PCR) analysis of relative transcript expression of 18 candidate genes encoding $S s D C L, S s A G O$, and $S s R D R$ in $S$. spontaneum clone SES208 exposed to dehydration treatment (PEG-6000) for 0, 3, 6, and $12 \mathrm{~h}$. The $\mathrm{x}$-axis indicates the time points of PEG-6000 exposure, whereas the y-axis indicates the relative expression level. The top young leaves were sampled and used for qRT-PCR assay. Relative transcript expression values are presented as means \pm standard errors based on three biological replicates with three technical replicates. Significant differential expression is indicated by an asterisk $\left({ }^{*}, p<0.05 ;{ }^{* *}, p<0.01\right)$. 
challenges for producing a reference sequence ${ }^{23}$. Recently three sugarcane genomes, one S. spontaneum clone ${ }^{28}$ and two modern cultivars ${ }^{27,30}$ have been sequenced and assembled to provide valuable reference genomes for identification of gene families and functions in sugarcane. RNA silencing in plants plays an important role in regulating gene expression at different levels via sRNAs ${ }^{5}$. Thus, in this study we performed genome-wide analysis of three gene families involved in RNA silencing, DCL, AGO, and RDR in S. spontaneum, and further analyzed the expression profiles of these genes in response to biotic and abiotic stresses.

Genetic diversity and evolution of DCL, AGO, and RDR gene families in plants. To date, at least 20 plant species have been used to investigate the genetic diversity of the AGO, DCL, and RDR gene families, and in this study we confirmed that members of these families showed obviously species-specific variations (Table S5). The number of genes encoding DCLs varied among plant species from four AtDCLs in Arabidopsis thaliana ${ }^{21}$ to eight OsDCLs in rice ${ }^{44}$, millet ${ }^{47}$, and B. napus ${ }^{21}$. In S. spontaneum, we identified only three classes of DCL genes (DCL1/2/3) and found no sequences corresponding to the DCL4 gene. Similarly, a DCL4 gene was not identified in $B$. oleracea ${ }^{21}$ or $A$. duranensis ${ }^{48}$. The number of genes encoding AGOs ranged from seven in cucumber ${ }^{49,50}$ to 27 in B. napus ${ }^{21}$. Total 21 SsAGO genes were identified in S. spontaneum. Notably, the monocot plants including rice, maize, and sugarcane have evolved an AGO18 subclade that falls into the AGO1/5/10 clade $^{51,52}$. The minimum number (5) of RDR gene family members was found in seven plants including rice ${ }^{44}$, maize $^{39}$, grapevine ${ }^{53}$, and three legume crops (chickpea, pigeonpea, and groundnut) ${ }^{48}$, whereas $B$. napus had the maximum number $(16)^{21}$. Our findings revealed that DCL4 and AGO1, which are commonly seen in both monocot and dicot plants, were lost in S. spontaneum, suggesting that the presence of novel DCL4- and AGO1like genes are substitute for the functions of the two genes in S. spontaneum.

Large segmental duplications and/or tandem duplications might be responsible for evolution of these three gene families, especially for AGOs in rice, giving rise to redundancy or contrasting functions among OsAGO genes $^{44}$. For example, large segmental duplications occurred in OsDCL2a-2b, OsAGO1a-1b, and OsAGO13-14, which localized on different chromosomes in rice. On the other hand, tandem duplications were present in three OsAGO gene pairs, OsAGO4a-15, OsAGO2-3, and OsAGO11-12, which localized close to each other on chromosomes 1,4 , and 3, respectively. Notably, large segmental duplication events commonly occurred in the AGOs, DCL, and RDR gene families in S. spontaneum, suggesting segmental duplication was an important evolution force for three gene families. However, segmental duplication did not appear in the OsDCL1 or OsRDR genes in rice ${ }^{44}$. Tandem duplication was also another important evolutionary force during evolution of S. spontaneum $A G O$ genes, but not for OsDCLs or OsRDRs. Similarly, no tandem duplication events occurred among OsDCLs and OsRDRs in rice ${ }^{44}$.

Different RDR-DCL-AGO combinations are synergistically involved in specialized RNA silencing to control invading nucleic acids from endogenous (mainly transposons) or exogenous (mainly viruses) origins, and are mediated by diverse sRNAs such as miRNA, transacting siRNAs (ta-siRNA), natural-antisense-transcript-siRNA (nat-siRNA) and virus-derived siRNAs (vsiRNA) ${ }^{13,54,55}$. For instance, DCL1 and AGO1 mainly participate in the miRNA pathway, but the RDR protein is not necessary for miRNA biogenesis ${ }^{56}$, RDR6, SGS3, AGO1 and DCL4 are the main components of the ta-siRNA pathway at the post-transcriptional level $l^{57}$, RDR2, AGO4, and DCL3 are involved in the RNA-directed DNA methylation (RdDM) pathway ${ }^{58}$, DCL2/3/4 and RDR6 are common essential components in antiviral RNA silencing medicated by vsiRNA in Arabidopsis and rice, while HSP90-activated AGO1/2/4/5/7/10 is loaded with a vsiRNA in Arabidopsis but AGO18 positively regulates AGO1 binding to vsiRNAs by sequestering miR168 in rice ${ }^{51}$. In this study, PPI network analysis also indicated that these core component interactions may participate in various RNA silencing pathways. Notably, the SsAGO18s representing a distinct AGO subfamily specific to monocots also actively interacted with two other components.

Response of DCL gene family members to drought and bacterial stresses. Four types of Dicer or DCL proteins are key components in miRNA and siRNA biogenesis pathways and mediate conversion of long double-stranded RNAs into mature small RNAs ${ }^{5,59}$. These DCLs (DCL1/2/3/4) play different roles in RNA interference-related processes of small RNA biogenesis in A. thaliana: DCL1 produces miRNAs and trigger post-transcriptional gene silencing (PTGS), DCL2 is essential for secondary siRNA-mediated transitive silencing via production of some virus-derived siRNAs; DCL3 produces endogenous RDR2-dependent siRNAs; and DCL4 functions in antiviral defense and development pathways by processing ta-siRNA precursors in the small RNA biogenesis pathway ${ }^{59-61}$. However, DCL2, DCL3, and DCL4 showed functional redundancy in siRNA and tasiRNA production ${ }^{62,63}$.

Here, we found that PEG treatment induced substantial upregulation of SsDCL1a, SsDCL2, and SsDCL3, particularly in $S s D C L 2$ and $S s D C L 3$. These findings are similar to those seen for $Z m D C L 2 / 3 b$ in maize ${ }^{39,64}$ and CaDCL1/2/3 in pepper ${ }^{64}$ that showed moderately and significantly, respectively, upregulated expression levels in response to drought conditions. Meanwhile, in the presence of X. albilineans infection, SsDCL2 and SsDCL3 expression levels were upregulated but SsDCL1a was unchanged or even downregulated. Similarly, in Brassica, the $B n D C L 1 a$ gene was downregulated to varying degrees at 8 and 16 hpi with Sclerotinia sclerotiorum ${ }^{21}$. However, previous studies suggested that DCL1-generated sRNAs can positively regulate antibacterial and antifungal immunity ${ }^{13}$. In addition, a previous study showed that levels of $S L D C L 1 / 2 a / 2 c / 2 d / 3$ were significantly upregulated in tomato plants infected with Tomato yellow leaf curl virus ${ }^{65}$. In pepper, CaDCL2/3/4 genes were highly expressed following infection with Cucumber mosaic virus (CMV) and particularly with Potato virus Y (PVY) or Tobacco mosaic virus (TMV) ${ }^{64}$. These findings suggested that DCL2 and DCL3 play positive roles in the response of plants to pathogen infection and abiotic stress, but the role of DCL1 may vary depending on plant species or the nature of biotic and abiotic stresses. 


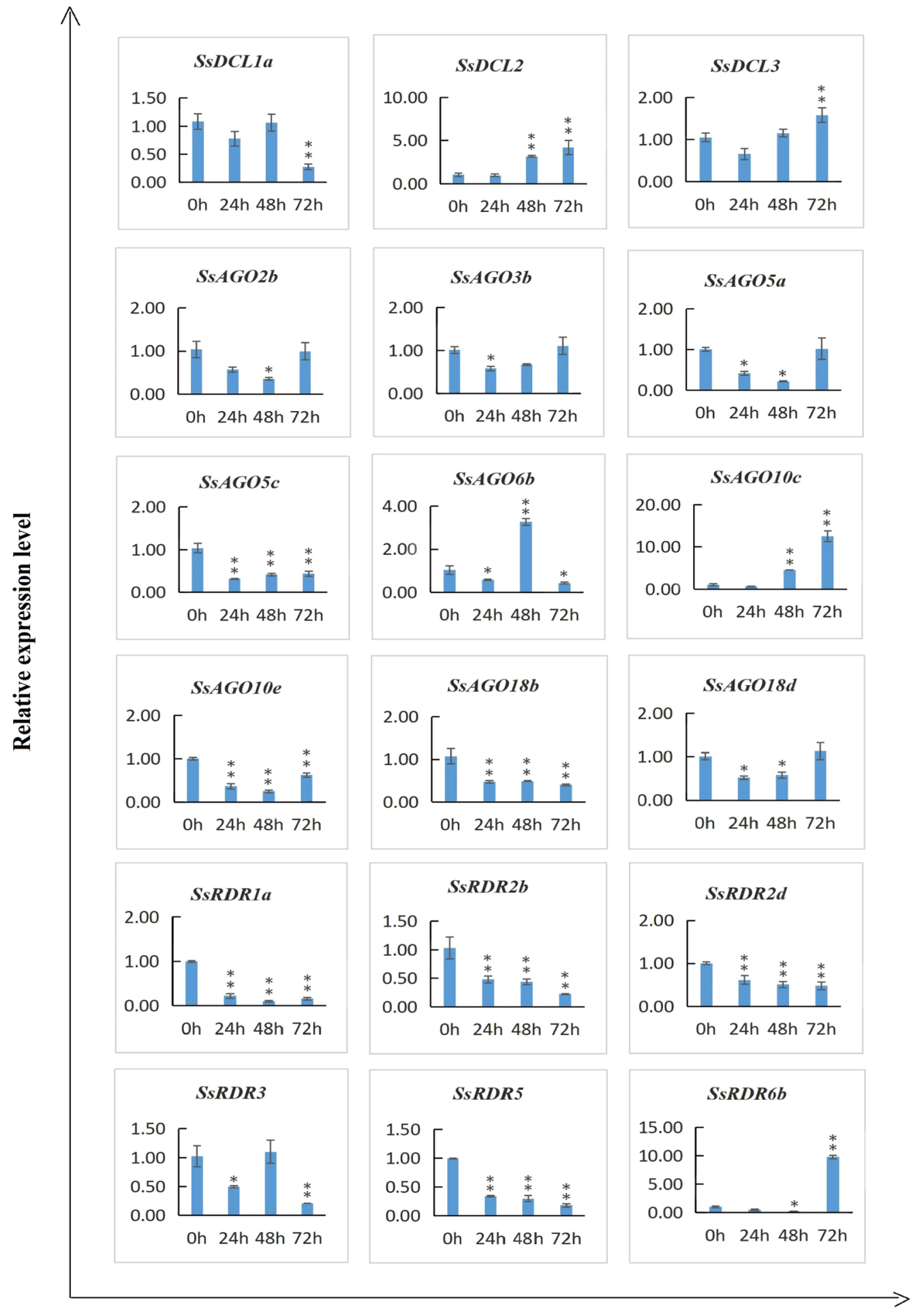

Figure 8. Quantitative real time PCR (qRT-PCR) analysis of relative transcript expression of 18 candidate genes encoding $S s D C L, S s A G O$, and $S s R D R$ in S. spontaneum clone SES208 inoculated with X. albilineans strain Xa-FJ1 at 0, 24, 48, and $72 \mathrm{~h}$ post-infection (hpi). The $\mathrm{X}$-axis indicates the time points of experimental treatment, while the $y$-axis indicates the relative expression level. The top young leaves were sampled and used for qRT-PCR assay. Relative transcript expression values are presented as means \pm standard errors based on three biological replicates with three technical replicates. Significant differential expression is indicated by an asterisk $\left({ }^{*}, p<0.05 ;{ }^{* *}, p<0.01\right)$. 
AGO gene family responses to drought and bacterial stress. AGOs together with small RNAs participate in RNA-induced silencing via cleavage of target mRNAs or blocking their translation ${ }^{66}$. Of the ten AtAGO family members in Arabidopsis, AGO2 regulates antibacterial immunity by binding miR393b ${ }^{*}$ to modulate exocytosis of antimicrobial PR proteins via a Golgi-localized SNARE gene MEMB12 ${ }^{67}$. AtAGO3 primarily binds 24-nt sRNAs with $5^{\prime}$-terminal adenines ${ }^{68}$. AtAGO5 can bind both viral RNAs and microRNAs to control plant-microbe interactions and plant physiology such as regulation of systemic resistance of Arabidopsis against Potato virus $X^{69}$. AtAGO6 is involved in siRNA accumulation, RdDM and transcriptional gene silencing ${ }^{70,71}$ whereas AtAGO10 promotes miR165/6 degradation via SDN1 and SDN2 exonucleases ${ }^{72}$. AGO18s is unique to monocots and in rice confers broad-spectrum virus resistance by sequestering host miR168 (microRNA168) or miR528 (microRNA528) following viral infection ${ }^{73,74}$.

Among the nine SsAGOs genes identified in this study, transcript levels of five genes (SsAGO2b/5a/5c/6b/10c) especially in SsAGO10c were highly increased in S. spontaneum under dehydration stress. Similar results were reported for genes in other plants subjected to drought stress, such as SlAGO6 in tomato ${ }^{65}$, PtAGO5b gene in poplar $^{75}$, and $C a A G O 2$ and $C a A G O 10 b$ in pepper ${ }^{64}$. Qin et al. (2018) suggested that $C a A G O 10 b$ might response to osmotic stress of pepper plants by regulating ABA (abscisic acid) responsive genes ${ }^{64}$. On the other hand, expression levels of all the tested $S s A G O$ genes, expect for $S s A G O 6 b / 10 c$, were significantly depressed to some extent in S. spontaneum plants after X. albilineans infection. Conversely, among the ten AGOs in Arabidopsis, only AGO2 is induced by bacterial infection and $\mathrm{AGO} 2$ positively regulates immunity by protein arginine methyltransferase 5 (PRMT5)-mediated dual regulation of this protein as well as associated sRNA levels to ensure appropriate plant immune responses ${ }^{76}$. A study by Qin et al. ${ }^{64}$ revealed that expression of CaAGO6, and the CaAGO10b gene in particular, was obviously upregulated in pepper upon inoculation with TMV, CMV, or PVY.

Notably, one gene pair, SsAGO10c/10e, which had a tandem duplication, exhibited different transcript expression patterns for PEG-treatment and $X$. albilineans infection, indicating that these genes may have evolved by segmental duplication events that were followed by differentiation of expression patterns. Kapoor et al. ${ }^{44}$ also proposed that the expression patterns of most OsAGO genes differentiated before their evolution by duplication events (tandem or segmental). For the unique class of AGO18 in monocot plants, the expression of $S s A G O 18 b$ and SsAGO18d was significantly depressed in S. spontaneum exposed to PEG-treatment stress or X. albilineans infection, suggesting these two genes may play a negative role in the response of $S$. spontaneum to biotic or abiotic stresses. However, a previous study showed that AGO18s confers positive regulation in broad-spectrum virus resistance in rice ${ }^{73,74}$. This constricting result may be due to the AGO18s from different plants playing different mechanisms, but additional investigation is needed to determine what roles this protein confers in sugarcane resistance to stress. Very little is known about AGO18 gene functions in monocot plants in response to abiotic stress.

Response of the RDR gene family to drought and bacterial stresses. RDRs play an important role in vsRNA biogenesis and vsRNA-mediated antiviral defenses in plants ${ }^{77}$. Arabidopsis has six AtRDRs, and at least three types act in distinct biological processes such as viral defense and chromatin silencing ${ }^{44}$. Furthermore, binding of transcription factors to the promoters of RDR1-6 genes may play important roles in how various plant species respond to biotic stresses ${ }^{78}$. Our data revealed that expression of three RDR genes, $S s R D R 1 a / 2 d / 5$, was significantly downregulated, but two $(S s R D R 3 / 6 b)$ were upregulated and one gene (SsRDR2b) was not significantly affected by PEG-treatment stress. In contrast to our results, levels of SIRDR1 in tomato ${ }^{65}$, PtRDR1c/1d in poplar ${ }^{75}$, and CaRDR1 in pepper ${ }^{64}$ were strongly increased by PEG-treatment stress. Upon X. albilineans infection, $S s R D R 1 a / 2 b / 5$ gene expression was significantly downregulated, but the $S s R D R 6 b$ gene was strongly upregulated.

Our findings suggested that SsRDR6 plays an important role in defenses against biotic and abiotic stresses in $S$. spontaneum. Similar results were seen for pepper, in which CaRDR6 gene expression levels were highly increased in the presence of three biotic stresses (CMV, PVY, and TMV inoculation) ${ }^{64}$. In contrast, Arabidopsis RDR6 acts as a novel negative regulator of pattern-triggered immunity (PTI) and an $r d r 6$ mutant exhibits enhanced basal resistance towards a virulent Pseudomonas syringae strain ${ }^{79}$. Previous observations showed that RDR6 in Nicotiana attenuata played an important role when plants respond to challenges in their native environments ${ }^{80}$. In rice, OsRDR6 is responsible for the observed ABA (abscisic acid)-mediated amplification and silencing of RDR6-dependent siRNA transcripts ${ }^{81}$.

\section{Conclusions}

In this study we identified 21 SsAGOs, four SsDCLs and 11 SsRDRs in the S. spontaneum genome. Genes in these three families present characteristic conserved domains and cis-elements as well as distinct expression profiles. Chromosome localization analysis revealed that segmental and/or tandem duplication contributed to the evolution of these genes, particularly for the SsAGO family. RNA-seq data analysis indicated tissue-specific expression patterns for some genes such as $S s R D R 5$, which showed preferential expression in leaves, whereas $S s A G O 18 c, S s A G O 18 b, S s A G O 10 e$, and $S s A G O 6 b$ exhibited stem-specific expression. Additionally, qRT-PCR analysis indicated that the expression patterns of some genes in three families differed in $S$. spontaneum plants exposed to PEG-treatment stress or X. albilineans infection in that $S s A G O 10 c$, SsDCL2, and SsRDR6b genes were strongly upregulated and $S s A G O 18 b, S s R D R 1 a, S s R D R 2 b / 2 d$, and $S s R D R 5$ were significantly depressed under the two stresses. Our findings can enhance our knowledge of the roles of these genes encoding SsAGOs, SsDCLs, and SsRDRs in biotic and abiotic stress response of sugarcane plants.

Data availability

All data, including image files, are available from the corresponding author on reasonable request. 
Received: 22 January 2020; Accepted: 23 July 2020

Published online: 06 August 2020

\section{References}

1. Chen, X. Small RNAs and their roles in plant development. Annu. Rev. Cell. Dev. Biol. 25, 21-44 (2009).

2. Lopez-Gomollon, S. \& Dalmay, T. Recent patents in RNA silencing in plants: constructs, methods and applications in plant biotechnology. Recent Pat. DNA Gene Seq. 4, 155-166 (2010).

3. Sarkies, P. \& Miska, E. A. Small RNAs break out: the molecular cell biology of mobile small RNAs. Nat. Rev. Mol. Cell Biol. 15, 525-535 (2014).

4. Valencia-Sanchez, M. A., Liu, J., Hannon, G. J. \& Parker, R. Control of translation and mRNA degradation by miRNAs and siRNAs. Genes Dev. 20, 515-524 (2006).

5. Axtell, M. J. Classification and comparison of small RNAs from plants. Annu. Rev. Plant Biol. 64, 137-159 (2013).

6. Hutvagner, G. \& Simard, M. J. Argonaute proteins: key players in RNA silencing. Nat. Rev. Mol. Cell Biol. 9, 22-32 (2008).

7. Carbonell, A. Plant ARGONAUTEs: Features, functions, and unknowns. Methods Mol. Biol. 1640, 1-21 (2017).

8. David, B. RNA silencing in plants. Nature 431, 356-363 (2004).

9. Guo, Z., Li, Y. \& Ding, S. W. Small RNA-based antimicrobial immunity. Nat. Rev. Immunol. 19, 31-44 (2019).

10. Voinnet, O. Use, tolerance and avoidance of amplified RNA silencing by plants. Trends Plant Sci. 13, 317-328 (2008).

11. Hunter, L. J. et al. RNA-dependent RNA polymerase 1 in potato (Solanum tuberosum) and its relationship to other plant RNAdependent RNA polymerases. Sci. Rep. 6, 23082 (2016).

12. Hua, C., Zhao, J. H. \& Guo, H. S. Trans-kingdom RNA silencing in plant-fungal pathogen interactions. Mol. Plant 11, 235-244 (2018)

13. Huang, C. Y., Wang, H., Hu, P., Hamby, R. \& Jin, H. Small RNAs big players in plant-microbe interactions. Cell Host Microbe 26, 173-182 (2019).

14. Zhu, C., Liu, T., Chang, Y. N. \& Duan, C. G. Small RNA functions as a trafficking effector in plant immunity. Int. J. Mol. Sci. 20, 2816 (2019).

15. Liu, Q., Feng, Y. \& Zhu, Z. Dicer-like (DCL) proteins in plants. Funct. Integr. Genomics 9, 277-286 (2009).

16. Fukudome, A. \& Fukuhara, T. Plant dicer-like proteins: double-stranded RNA-cleaving enzymes for small RNA biogenesis. J. Plant. Res. 130, 33-44 (2017).

17. Tolia, N. H. \& Joshua-Tor, L. Slicer and the argonautes. Nat. Chem. Biol. 3, 36-43 (2007)

18. Mallory, A. \& Vaucheret, H. Form, function, and regulation of ARGONAUTE proteins. Plant Cell 22, 3879-3889 (2010).

19. Willmann, M. R., Endres, M. W., Cook, R. T. \& Gregory, B. D. The functions of RNA-dependent RNA polymerases in Arabidopsis. Arabidopsis Book 9, e146 (2011).

20. Vazquez, F. Arabidopsis endogenous small RNAs: Highways and byways. Trends Plant Sci. 11, 460-468 (2011).

21. Cao, J. Y. et al. Genome-wide identification of dicer-Like, Argonaute, and RNA-dependent RNA polymerase gene families in Brassica species and functional analyses of their Arabidopsis homologs in resistance to Sclerotinia sclerotiorum. Front. Plant Sci. 7, 1614 (2016).

22. Ali, A., Khan, M., Sharif, R., Mujtaba, M. \& Gao, S.J. Sugarcane Omics: An update on the current status of research and crop improvement. Plants (Basel) 8, 344 (2019).

23. Diniz, A. L. et al. Genomic resources for energy cane breeding in the post genomics era. Comput. Struct. Biotechnol. J. 17, 1404-1414 (2019).

24. D'Hont, A. et al. Characterisation of the double genome structure of modern sugarcane cultivars (Saccharum spp.) by molecular cytogenetics. Mol. Gen. Genet. 250, 405-413 (1996).

25. Zhou, M. Sugarcane (S. Officinarum X S. Spontaneum). In: Campos H, Caligari PDS, eds. Genetic Improvement of Tropical Crops. 291-308 (Springer, Cham, 2017).

26. Moore, P. H., Nagai, C. \& Fitch, M. Production and evaluation of sugarcane haploids. Proc. Int. Soc. Sugar Cane Technol. 20, 599-607 (1989).

27. Garsmeur, O. et al. A mosaic monoploid reference sequence for the highly complex genome of sugarcane. Nat. Commun. 9, 2638 (2018).

28. Zhang, J. et al. Allele-defined genome of the autopolyploid sugarcane Saccharum spontaneum L. Nat. Genet. 50, 1565-1573 (2018).

29. Xu, F. et al. Comparative analysis of two sugarcane ancestors Saccharum officinarum and S. spontaneum based on complete chloroplast genome sequences and photosynthetic ability in cold stress. Int. J. Mol. Sci. 20, 3828 (2019).

30. Souza, G. M. et al. Assembly of the $373 \mathrm{k}$ gene space of the polyploid sugarcane genome reveals reservoirs of functional diversity in the world's leading biomass crop. Gigascience 8, 12 (2019).

31. Gao, S., Luo, J., Zhang, H., Chen, R. \& Lin, Y. Physiological and biochemical indexes of drought resistance of sugarcane (Saccharum spp.). Ying Yong Sheng Tai Xue Bao 17, 1051-1054 (2006).

32. McQualter, R. \& Dookun-Saumtally, A. Expression profiling of abiotic-stress-inducible genes in sugarcane. Proc. Int. Soc. Sugar cane Technol. 29, 878-888 (2007).

33. Royer, M., Pieretti, I., Cociancich, S. \& Rott, P. Recent progress in understanding three major bacterial diseases of sugarcane: Gumming, leaf scald and ratoon stunting. In: Rott, P., eds. Achieving Sustainable Cultivation of Sugarcane, Breeding, Pests and Diseases (2nd ed.) 311-336 (Burleigh Dodds Science Publishing, Cambridge, 2018).

34. Lin L-H. et al. Molecular detection and prevalence of Xanthomonas albilineans, the causal agent of sugarcane leaf scald, in China. Plant Protect. 109, 17-23 (2018).

35. Ntambo, M. S. et al. Identification and characterization of Xanthomonas albilineans causing sugarcane leaf scald in China using multilocus sequence analysis. Plant Pathol. 68, 269-277 (2019).

36. Finn, R. D. et al. The Pfam protein families database: towards a more sustainable future. Nucleic Acids Res. 44, D279-D285 (2016).

37. Finn, R. D., Clements, J. \& Eddy, S. R. HMMER web server: Interactive sequence similarity searching. Nucleic Acids Res. 39, W29-W37 (2011)

38. Xie, T. et al. Genome-wide investigation of WRKY gene family in pineapple: evolution and expression profiles during development and stress. BMC Genomic 19, 490 (2018).

39. Qian, Y. et al. Identification and characterization of Dicer-like, Argonaute and RNA-dependent RNA polymerase gene families in maize. Plant Cell Rep. 30, 1347-1363 (2011).

40. Hu B. et al. GSDS 2.0: An upgraded gene feature visualization server. Bioinformatics 31, 1296-1297 (2015).

41. Szklarczyk, D. et al. STRING v11: Protein-protein association networks with increased coverage, supporting functional discovery in genome-wide experimental datasets. Nucleic Acids Res. 47, D607-D613 (2019)

42. Krzywinski, M. et al. Circos: An information aesthetic for comparative genomics. Genome Res. 19, 1639-1645 (2009).

43. Wang, Y. et al. MCScanX: A toolkit for detection and evolutionary analysis of gene synteny and collinearity. Nucleic Acids Res. 40, e49 (2012)

44. Kapoor, M. et al. Genome-wide identification, organization and phylogenetic analysis of dicer-like, Argonaute and RNA-dependent RNA polymerase gene families and their expression analysis during reproductive development and stress in rice. BMC Genomics 9, 451 (2008). 
45. Kumar, S., Stecher, G., Li, M., Knyaz, C. \& Tamura, K. MEGA X: molecular evolutionary genetics analysis across computing platforms. Mol. Biol. Evol. 35, 1547-1549 (2018).

46. Livak, K. J. \& Schmittgen, T. D. Analysis of relative gene expression data using real-time quantitative PCR and the 2(-Delta Delta $\mathrm{C}(\mathrm{T})$ ) method. Methods 25, 402-408 (2001).

47. Yadav, C. B., Muthamilarasan, M., Pandey, G. \& Prasad, M. Identification, characterization and expression profiling of dicer-like, Argonaute and RNA-dependent RNA polymerase gene families in Foxtail millet. Plant Mol. Biol. Rep. 33, 43-55 (2015).

48. Garg, V. et al. Genome-wide identification, characterization, and expression analysis of small RNA biogenesis purveyors reveal their role in regulation of biotic stress responses in three legume crops. Front. Plant Sci. 8, 488 (2017).

49. Gan, D. et al. Expression analysis of argonaute, dicer-like, and RNA-dependent RNA polymerase genes in cucumber (Cucumis sativus L.) in response to abiotic stress. J. Genet. 96, 235-249 (2017).

50. Gan D. F. et al. Genome-wide identification of the dicer-like, Argonaute, and RNA-dependent RNA polymerase gene families in cucumber (Cucumis sativus L.). J. Plant Growth Regul. 35, 135-150 (2016).

51. Zhang, H., Xia, R., Meyers, B. C. \& Walbot, V. Evolution, functions, and mysteries of plant ARGONAUTE proteins. Curr. Opin. Plant Biol. 27, 84-90 (2015).

52. Fang, X. \& Qi, Y. RNAi in plants: An argonaute-centered view. Plant Cell. 28, 272-285 (2016).

53. Zhao, H. et al. Comprehensive analysis of dicer-like, Argonaute, and RNA-dependent RNA polymerase gene families in grapevine (Vitis Vinifera). J. Plant Growth Regul. 34, 108-121 (2014).

54. Brodersen, P. \& Voinnet, O. The diversity of RNA silencing pathways in plants. Trends Genet. 22, 268-280 (2006).

55. Borges, F. \& Martienssen, R. The expanding world of small RNAs in plants. Nat. Rev. Mol. Cell Biol. 16, 727-741 (2015)

56. Bologna, N. \& Voinnet, O. The diversity, biogenesis, and activities of endogenous ssilencing small RNAs in Arabidopsis. Annu. Rev. Plant. Biol. 65, 473-503 (2014).

57. Skopelitis, D., Husbands, A. \& Timmermans, M. Plant small RNAs as morphogens. Curr. Opin. Cell Biol. 24, 217-224 (2011).

58. Julie A, L. \& Steven E, J. Establishing, maintaining and modifying DNA methylation patterns in plants and animals. Nat. Rev. Genet. 11, 204-220 (2010).

59. Fukudome, A. \& Fukuhara, T. Plant dicer-like proteins: Double-stranded RNA-cleaving enzymes for small RNA biogenesis. J. Plant Res. 130, 33-44 (2017).

60. Kurihara, Y. \& Watanabe, Y. Arabidopsis micro-RNA biogenesis through dicer-like 1 protein functions. Proc. Natl. Acad. Sci. USA 101, 12753-12758 (2004).

61. Xie, Z., Allen, E., Wilken, A. \& Carrington, J. C. DICER-LIKE 4 functions in trans-acting small interfering RNA biogenesis and vegetative phase change in Arabidopsis thaliana. Proc. Natl. Acad. Sci. USA 102, 12984-12989 (2005).

62. Gasciolli, V., Mallory, A. C., Bartel, D. P. \& Vaucheret, H. Partially redundant functions of Arabidopsis DICER-like enzymes and a role for DCL4 in producing trans-acting siRNAs. Curr. Biol. 15, 1494-1500 (2005).

63. Henderson, I. et al. Dissecting Arabidopsis thaliana DICER function in small RNA processing, gene silencing and DNA methylation patterning. Nat. Genet. 38, 721-725 (2006).

64. Qin, L., Mo, N., Muhammad, T. \& Liang, Y. Genome-wide analysis of DCL, AGO, and RDR gene families in Pepper (Capsicum Annuum L.). Int. J. Mol. Sci. 19, 1038 (2018).

65. Bai, M. et al. Genome-wide identification of dicer-like, Argonaute and RNA-dependent RNA polymerase gene families and their expression analyses in response to viral infection and abiotic stresses in Solanum lycopersicum. Gene 501, 52-62 (2012).

66. Ma, Z. \& Zhang, X. Actions of plant Argonautes: Predictable or unpredictable?. Curr. Opin. Plant Biol. 45, 59-67 (2018).

67. Zhang, X. et al. Arabidopsis Argonaute 2 regulates innate immunity via miRNA393*-mediated silencing of a golgi-localized SNARE gene, MEMB12. Mol. Cell. 42, 356-366 (2011).

68. Zhang, Z., Liu, X., Guo, X., Wang, X. J. \& Zhang, X. Arabidopsis AGO3 predominantly recruits 24-nt small RNAs to regulate epigenetic silencing. Nat. Plants 2, 16049 (2016).

69. Reyero-Saavedra, M. et al. Gene silencing of Argonaute5 negatively affects the establishment of the legume-rhizobia symbiosis. Genes (Basel) 8, 352 (2017).

70. Zheng, X., Zhu, J., Kapoor, A. \& Zhu, J. K. Role of Arabidopsis AGO6 in siRNA accumulation, DNA methylation and transcriptional gene silencing. EMBO. J. 26, 1691-1701 (2007).

71. Duan, C. G. et al. Specific but interdependent functions for Arabidopsis AGO4 and AGO6 in RNA-directed DNA methylation. EMBO. J. 34, 581-592 (2015).

72. Yu, Y. et al. ARGONAUTE10 promotes the degradation of miR165/6 through the SDN1 and SDN2 exonucleases in Arabidopsis. PLoS Biol. 15, e2001272 (2017).

73. Wu, J. et al. Viral-inducible Argonaute 18 confers broad-spectrum virus resistance in rice by sequestering a host microRNA. Elife. 4, e05733 (2015).

74. Wu, J. et al. ROS accumulation and antiviral defence control by microRNA528 in rice. Nat. Plants 3, 16203 (2017).

75. Zhao, K. et al. The dicer-like, Argonaute and RNA-dependent RNA polymerase gene families in Populus trichocarpa: gene structure, gene expression, phylogenetic analysis and evolution. J. Genet. 94, 317-321 (2015).

76. Hu, P. et al. Dual regulation of Arabidopsis AGO2 by arginine methylation. Nat. Commun. 10, 844 (2019).

77. Qi, X., Bao, F. S. \& Xie, Z. Small RNA deep sequencing reveals role for Arabidopsis thaliana RNA-dependent RNA polymerases in viral siRNA biogenesis. PLoS ONE 4, e4971 (2009).

78. Prakash, V. \& Chakraborty, S. Identification of transcription factor binding sites on promoter of RNA dependent RNA polymerases (RDRs) and interacting partners of RDR proteins through In-silico analysis. Physiol. Mol. Biol. Plants 25, 1055-1071 (2019).

79. Boccara, M. et al. The Arabidopsis miR472-RDR6 silencing pathway modulates PAMP- and effector-triggered immunity through the post-transcriptional control of disease resistance genes. PLoS Pathog. 10, e1003883 (2014).

80. Shree, P., Emmanuel, G., Klaus, G. \& Ian, T. RNA-directed RNA polymerase 3 from Nicotiana Attenuata is required for competitive growth in natural environments. Plant Physiol. 147, 1212-1224 (2008).

81. Ji, H. Y. et al. Phytohormone abscisic acid control RNA-dependent RNA polymerase 6 gene expression and post-transcriptional gene silencing in rice cells. Nucleic Acids Res. 36, 1220-1226 (2008).

\section{Acknowledgements}

This research was funded by the earmarked fund for the China Agricultural Research System (grant no. CARS170302), and the special fund for science and technology innovation of the Fujian Agriculture and Forestry University, China (grant no. CXZX2018024).

\section{Author contributions}

D.L.C carried out analysis of the results and preparation of a draft manuscript; J.Y.M., X.Y.R., and J.J.Y. performed preparation of plant materials and experimental treatments. H.Y.F. and M.T.H. participated in some experiments. Q.Q.Z. and S.J.G. conceived and supervised the project, and writing and finalizing of the manuscript. All authors read and approved the final manuscript. 


\section{Competing interests}

The authors declare no competing interests.

\section{Additional information}

Supplementary information is available for this paper at https://doi.org/10.1038/s41598-020-70061-7.

Correspondence and requests for materials should be addressed to Q.-Q.Z. or S.-J.G.

Reprints and permissions information is available at www.nature.com/reprints.

Publisher's note Springer Nature remains neutral with regard to jurisdictional claims in published maps and institutional affiliations.

Open Access This article is licensed under a Creative Commons Attribution 4.0 International License, which permits use, sharing, adaptation, distribution and reproduction in any medium or format, as long as you give appropriate credit to the original author(s) and the source, provide a link to the Creative Commons license, and indicate if changes were made. The images or other third party material in this article are included in the article's Creative Commons license, unless indicated otherwise in a credit line to the material. If material is not included in the article's Creative Commons license and your intended use is not permitted by statutory regulation or exceeds the permitted use, you will need to obtain permission directly from the copyright holder. To view a copy of this license, visit http://creativecommons.org/licenses/by/4.0/.

(C) The Author(s) 2020 